\title{
Event Horizon Telescope observations of the jet launching and collimation in Centaurus $\mathrm{A}$
}

\author{
Michael Janssen ${ }^{1,2} \llbracket$, Heino Falcke², Matthias Kadler ${ }^{3}$, Eduardo Ros ${ }^{\circledR 1}$, Maciek Wielgus ${ }^{4,5}$, \\ Kazunori Akiyama ${ }^{4,6,7}$, Mislav Balokovićc, ${ }^{8,9}$, Lindy Blackburn (10) 4,5, Katherine L. Bouman (1) 4,5,10, \\ Andrew Chael ${ }^{11}$, Chi-kwan Chan ${ }^{12,13}$, Koushik Chatterjee ${ }^{14}$, Jordy Davelaar ${ }^{2,15,16}$, \\ Philip G. Edwards ${ }^{17}$, Christian M. Fromm ${ }^{4,5,18}$, José L. Gómez ${ }^{119}$, Ciriaco Goddi ${ }^{2,20}$, \\ Sara Issaoun², Michael D. Johnson ${ }^{4,5}$, Junhan Kim ${ }^{10,12}$, Jun Yi Koay ${ }^{21}$, Thomas P. Krichbaum ${ }^{1}$, \\ Jun Liu', Elisabetta Liuzzo (1) ${ }^{22}$, Sera Markoff ${ }^{14,23}$, Alex Markowitz ${ }^{24}$, Daniel P. Marrone (i) ${ }^{12}$, \\ Yosuke Mizuno $\mathbb{1}^{18,25}$, Cornelia Müller ${ }^{1,2}$, Chunchong Ni ${ }^{26,27}$, Dominic W. Pesce ${ }^{4,5}$, \\ Venkatessh Ramakrishnan ${ }^{28}$, Freek Roelofs ${ }^{2,5}$, Kazi L. J. Rygl (iD ${ }^{22}$, Ilse van Bemmel (iD ${ }^{29}$ and \\ The Event Horizon Telescope Collaboration*
}

\begin{abstract}
Very-long-baseline interferometry (VLBI) observations of active galactic nuclei at millimetre wavelengths have the power to reveal the launching and initial collimation region of extragalactic radio jets, down to 10-100 gravitational radii $\left(r_{g} \equiv G M / c^{2}\right)$ scales in nearby sources ${ }^{1}$. Centaurus $A$ is the closest radio-loud source to Earth ${ }^{2}$. It bridges the gap in mass and accretion rate between the supermassive black holes (SMBHs) in Messier 87 and our Galactic Centre. A large southern declination of $-43^{\circ}$ has, however, prevented VLBI imaging of Centaurus $A$ below a wavelength of $1 \mathrm{~cm}$ thus far. Here we show the millimetre VLBI image of the source, which we obtained with the Event Horizon Telescope at $228 \mathrm{GHz}$. Compared with previous observations ${ }^{3}$, we image the jet of Centaurus $A$ at a tenfold higher frequency and sixteen times sharper resolution and thereby probe sub-lightday structures. We reveal a highly collimated, asymmetrically edge-brightened jet as well as the fainter counterjet. We find that the source structure of Centaurus A resembles the jet in Messier 87 on $\sim 500 r_{g}$ scales remarkably well. Furthermore, we identify the location of Centaurus A's SMBH with respect to its resolved jet core at a wavelength of $1.3 \mathrm{~mm}$ and conclude that the source's event horizon shadow ${ }^{4}$ should be
\end{abstract}

visible at terahertz frequencies. This location further supports the universal scale invariance of black holes over a wide range of masses ${ }^{5,6}$.

Here we present the first image of Centaurus A (Cen A) obtained by the Event Horizon Telescope (EHT) with a nominal resolution of 25 microarcseconds ( $\mu \mathrm{as}$ ) at a wavelength $(\lambda)$ of $1.3 \mathrm{~mm}$. For a black hole mass of $(5.5 \pm 3) \times 10^{7} M_{\odot}\left(\right.$ ref. $\left.^{7}\right)$, we are probing jet structures down to scales of $\sim 200$ gravitational radii $r_{\mathrm{g}} \approx 0.6$ light days. It has recently become possible to model these scales with sophisticated general relativistic magnetohydrodynamics (GRMHD) simulations $^{8}$, where jet ejection and their symbiotic relationship with accretion flows are simulated from first principles. We have observed Cen A in a six-hour-long track on 10 April 2017. The EHT, as a novel and heterogeneous high-frequency very-long-baseline interferometry (VLBI) array, poses unique calibration challenges. To obtain robust results, independent of assumptions made during the data calibration, we base our scientific analysis on two datasets, which we obtained from two independent calibration pipelines: rPICARD' and EHT-HOPS ${ }^{10}$ ('Data reduction pipelines' in Methods).

Figure 1 presents our reconstruction of the jet image structure derived from the EHT data using a regularized maximum likelihood method, next to the large-scale source morphology and the

\footnotetext{
'Max-Planck-Institut für Radioastronomie, Bonn, Germany. ${ }^{2}$ Department of Astrophysics, Institute for Mathematics, Astrophysics and Particle Physics (IMAPP), Radboud University, Nijmegen, The Netherlands. ${ }^{3}$ Lehrstuhl für Astronomie, Universität Würzburg, Würzburg, Germany. ${ }^{4}$ Black Hole Initiative at Harvard University, Cambridge, MA, USA. ${ }^{5}$ Center for Astrophysics | Harvard \& Smithsonian, Cambridge, MA, USA. ${ }^{6}$ Massachusetts Institute of Technology Haystack Observatory, Westford, MA, USA. ${ }^{7}$ National Astronomical Observatory of Japan, Mitaka, Japan. ${ }^{8}$ Yale Center for Astronomy and Astrophysics, New Haven, CT, USA. ${ }^{9}$ Department of Physics, Yale University, New Haven, CT, USA. ${ }^{10}$ California Institute of Technology, Pasadena, CA, USA. "Princeton Center for Theoretical Science, Princeton University, Princeton, NJ, USA. ${ }^{2}$ Steward Observatory and Department of Astronomy, University of Arizona, Tucson, AZ, USA. ${ }^{13}$ Data Science Institute, University of Arizona, Tucson, AZ, USA. ${ }^{14}$ Anton Pannekoek Institute for Astronomy, University of Amsterdam, Amsterdam, The Netherlands. ${ }^{15}$ Department of Astronomy and Columbia Astrophysics Laboratory, Columbia University, New York, NY, USA. ${ }^{16}$ Center for Computational Astrophysics, Flatiron Institute, New York, NY, USA. ${ }^{17}$ Australia Telescope National Facility, CSIRO Astronomy and Space Science, Epping, New South Wales, Australia. ${ }^{18}$ Institut für Theoretische Physik, Goethe-Universität Frankfurt, Frankfurt, Germany. ${ }^{19}$ Instituto de Astrofísica de Andalucía-CSIC, Granada, Spain. ${ }^{20}$ Leiden Observatory-Allegro, Leiden University, Leiden, The Netherlands. ${ }^{21}$ Institute of Astronomy and Astrophysics, Academia Sinica, Taipei, Taiwan, ROC. ${ }^{22}$ Italian ALMA Regional Centre, INAF-Istituto di Radioastronomia, Bologna, Italy. ${ }^{23}$ Gravitation Astroparticle Physics Amsterdam (GRAPPA) Institute, University of Amsterdam, Amsterdam, The Netherlands. ${ }^{24}$ Nicolaus Copernicus Astronomical Center, Polish Academy of Sciences, Warszawa, Poland. ${ }^{25}$ Tsung-Dao Lee Institute and School of Physics and Astronomy, Shanghai Jiao Tong University, Shanghai, People's Republic of China. ${ }^{26}$ Department of Physics and Astronomy, University of Waterloo, Waterloo, Ontario, Canada. ${ }^{27}$ Waterloo Centre for Astrophysics, University of Waterloo, Waterloo, Ontario, Canada. ${ }^{28}$ Astronomy Department, Universidad de Concepción, Concepción, Chile. ${ }^{29}$ Joint Institute for VLBI ERIC (JIVE), Dwingeloo, The Netherlands. ${ }^{\star} A$ list of authors and their affiliations appears at the end of the paper. ${ }^{凶}$-mail: mjanssen@mpifr-bonn.mpg.de
} 


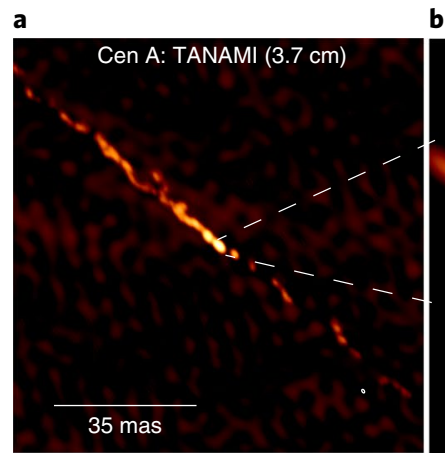
b
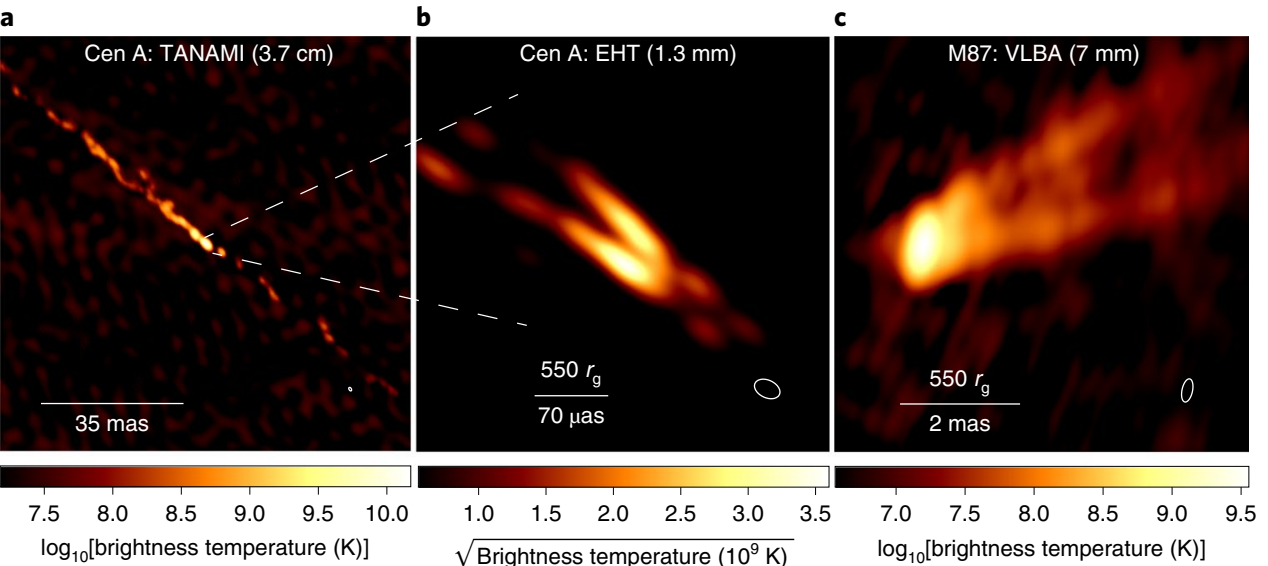

Fig. 1 | The jet structure of Cen A compared with M87. a, The large-scale jet of Cen A from an $8 \mathrm{GHz}(\lambda 3.7 \mathrm{~cm})$ TANAMI ${ }^{47}$ observation in November $2011^{3}$ on a logarithmic colour scale. b, Our final EHT image from April 2017, blurred to the nominal resolution for a uniform weighting of the visibilities. The reconstruction is based on the rPICARD data and is shown on a square-root scale, where values below a brightness temperature of $3 \times 10^{8} \mathrm{~K}$ are clipped, due to a lower dynamic range compared with the longer-wavelength observations. An unclipped and unconvolved version of this image is shown in Fig. 2. The dashed lines between $\mathbf{a}$ and $\mathbf{b}$ indicate the zoom-in of the EHT image with respect to the $\mathrm{cm} \mathrm{VLBI} \mathrm{jet.} \mathrm{c,} \mathrm{The} \mathrm{M87} \mathrm{jet} \mathrm{at} 43 \mathrm{GHz}(\lambda 7 \mathrm{~mm}$ ) from a Very Long Baseline Array (VLBA) observation in June $2013^{9,26}$ on a logarithmic scale. North is up and east is to the left. The physical, linear scales of the full field of views shown in the three images are 2 pc for TANAMI (a), 0.007 pc for the EHT (b) and 0.6 pc for the VLBA (c). The beams are shown in the bottom right corner of each image.

similarly edge-brightened morphology of the Messier 87 (M87) jet on comparable gravitational scales. These images are convolved with Gaussian beams set by their respective nominal instrumental resolutions, as per standard practice in radio-interferometric imaging, to suppress possibly spurious fine-scale structures in the image model. The brightness temperatures $\mathcal{T}(\mathrm{K})$ shown are related to flux densities $S$ in jansky (Jy) through the observing wavelength $\lambda$, Boltzmann constant $k_{\mathrm{B}}$ and angular resolution element $\Omega$ as $\mathcal{T}=\lambda^{2}\left(2 k_{\mathrm{B}} \Omega\right)^{-1} S$. The $\lambda 1.3 \mathrm{~mm}$ Cen A jet has a narrow, collimated profile and exhibits one-sidedness, pronounced edge-brightening and a northwest-southeast brightness asymmetry. The approaching jet extends towards the northeast and the faint counterjet is directed southwestwards. The total compact flux density in our image is $\sim 2$ Jy. The identification of the jet apex and black hole position ('The position of the jet apex' in Methods) is shown in the unconvolved image model of Fig. 2. We can use interferometer data with a high signal-to-noise ratio to super-resolve image features beyond the nominal resolution of the instrument. We therefore base our analysis on the robust features of the unconvolved image model. We have verified the robustness of the counterjet feature with synthetic data studies (Supplementary Fig. 1). The estimated jet position angle on the sky of $48^{\circ} \pm 5^{\circ}$ agrees with centimetre-wave VLBI observations ${ }^{3}$. The centimetre-band data also constrain the inclination angle of the jet axis with respect to our line of sight to $\theta \approx 12^{\circ}-45^{\circ}$, assuming that the jet does not bend along the line of sight.

The Cen A $\lambda 1.3 \mathrm{~mm}$ jet exhibits three types of brightness asymmetry $(\mathcal{R})$ : between the jet and counterjet, the sheath and spine, and the northwest versus southeast ridgelines ('Brightness asymmetries' in Methods). We take the two bright radiating streams of the approaching jet and counterjet as jet 'arms' and denote the maximum intensity region along each arm as 'ridgeline. The jet-to-counterjet intensity ratio $\mathcal{R}_{\mathrm{j} / \mathrm{cj}}$ can naturally be explained for a relativistic outflow with an inclination angle $\theta \neq 90^{\circ}$, where jet emission will be Doppler boosted and counterjet emission de-boosted. We find $\mathcal{R}_{\mathrm{j} / \mathrm{cj}} \gtrsim 5$, which is in agreement with centimetre-wave VLBI observations ${ }^{3}$ and suggests that the initial acceleration of the jet occurs within the inner collimation region imaged in this study.

There is no jet spine emission in our image. With synthetic data studies, we found that spine emission exceeding $\sim 20 \%$ of the sheath radiation intensity would be detectable, that is, $\mathcal{R}_{\text {sh/sp }}>5$ ('Synthetic data imaging tests' in Methods). The intensities of the brightest, central southeast and northwest jet components in the unconvolved image are $(32 \pm 8) \times 10^{9} \mathrm{~K}$ and $(20 \pm 4) \times 10^{9} \mathrm{~K}$, respectively. The brightness ratio between these components follows as $\mathcal{R}_{\mathrm{s} / \mathrm{n}}=1.6 \pm 0.5$.

The collimation profile of the jet width $W$ follows a narrow expansion profile with distance to the apex $z$ as $W \propto z^{k}$ with $k=0.33 \pm\left. 0.05\right|_{\text {stat }} \pm\left. 0.06\right|_{\text {sys }}$ (Fig. 3). Resolution and potentially optical depth effects prevent us from pinning down the jet opening angle $\psi_{\text {jet }}$ at small $z$, where the jet converges towards the apex. We denote the boundary between the inner convergence region and the outer jet with a clearly defined collimation and easily traceable jet ridgelines as $z_{\text {col }}$. For the brighter and straighter southeast arm, we have $W\left(z_{\text {col }} \approx 32 \mu\right.$ as $) \approx 25 \mu$ as, that is, the brightest jet component marks the boundary between the convergence and strongly collimated regions here (Fig. 2). If we assume the two jet ridgelines to meet at the apex, we find $\psi_{\text {jet }} \gtrsim 40^{\circ}$ as a conservative estimate. Factoring in the range of possible $\theta$ values yields $\psi_{\text {int }} \gtrsim 10^{\circ}-30^{\circ}$ for the intrinsic, deprojected opening angle ('Collimation profile' in Methods).

The $\mathrm{M}^{11} 7^{11}$ (NGC 4486, 3C 274, Virgo A), Markarian $501^{12}$ and restarted $3 \mathrm{C} 84$ jets $^{13}$ also show strong edge-brightening and large initial opening angles on comparable scales seen at similar inclination angles of $\sim 18^{\circ}$. The expansion profile of Cen A lies in between the parabolic profile of M87 $(k=0.5)$ and the almost cylindrical profile of $3 \mathrm{C} 84(k=0.2)$, which implies a strong confinement of the $3 \mathrm{C} 84$ jet by a shallow pressure gradient from the ambient medium. For the inner Cen A jet, this suggests strong magnetic collimation or the presence of external pressure and density gradients of $P_{\text {ext }} \propto z^{-4 k}=z^{-1.3}$ and $\rho_{\text {ext }} \propto z^{1-4 k}=z^{-0.3}$ ('Confinement by the ambient medium' in Methods). Radiatively inefficient accretion flows alone, which are expected to operate in the M87, 3C84 and Cen A sub-Eddington low-luminosity active galactic nuclei (LLAGN) sources, have comparatively steeper pressure and density gradients $^{14}$. This may indicate the presence of winds, which are likely to be launched by this type of accretion flow. The noticeable similarity and prominence of edge-brightened jet emission in M87, 3C84 and Cen A suggests the dominance of jet sheath emission to be an emerging feature in LLAGN. In GRMHD simulations, the sheath 
a

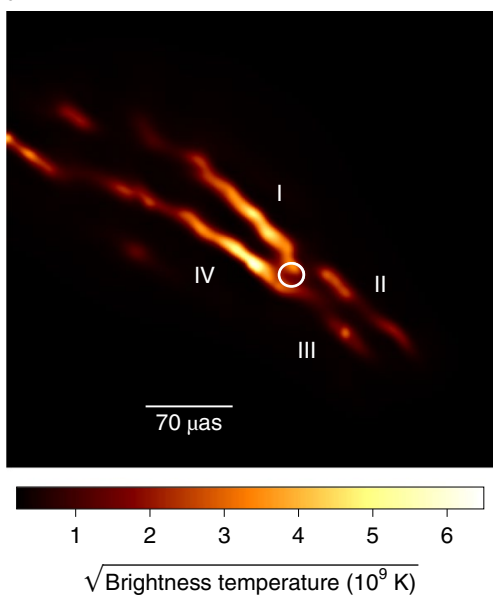

b

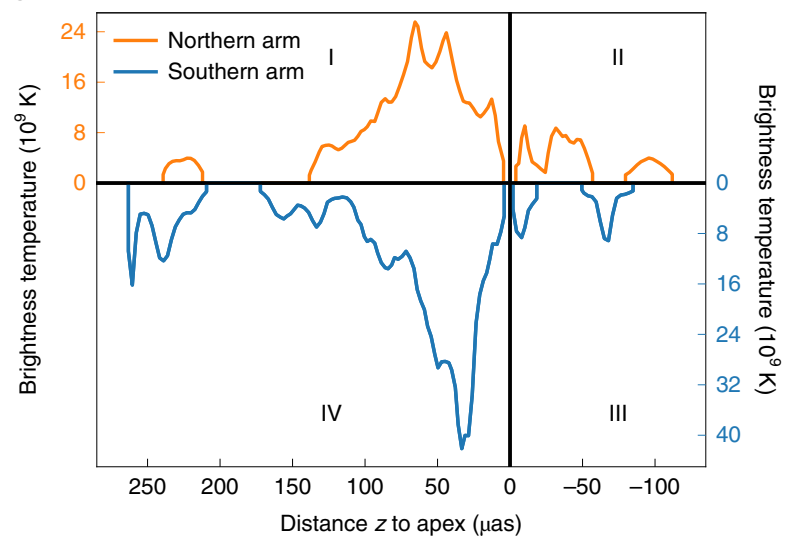

Fig. 2 | Image analysis of the final model. a, Model image corresponding to the image from Fig. 1 with a pixel size of $2 \mu$ as. Here, the tentative position of the jet apex is indicated with a circle. The size of the circle indicates the uncertainty in the apex location. $\mathbf{b}$, Central brightness temperatures along the jet ridgelines from the model in four quadrants as a function of distance to the jet apex. The quadrants I, II, III and IV correspond to the similarly marked regions of the jet in $\mathbf{a}$. Negative values for the distance to the jet apex are assigned for the counterjet region. Brightness temperatures of the fainter northwest (orange line) and brighter southeast (blue line) arms are shown in the top and bottom panels, respectively.

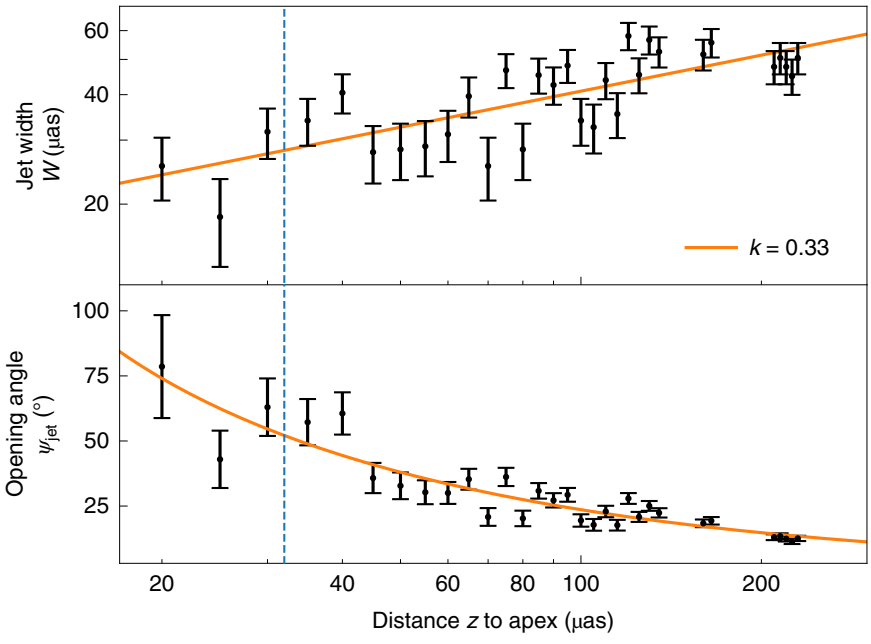

Fig. 3 | Cen A collimation profile derived from the final model. Top: the jet width $W$ as a function of apex distance $z$, overplotted with a $W(z)=A z^{k}$ least-squares fit, with $k=0.33 \pm\left. 0.05\right|_{\text {stat }} \pm\left. 0.06\right|_{\text {sys }}$ ('Collimation profile' in Methods). Here, $A$ is a nuisance parameter. The systematic error has been derived based on the uncertainty in the apex position. The error bars are derived from a $5 \mu$ as $1 \sigma$ statistical uncertainty on each pixel in the model. Bottom: the corresponding jet opening angle between the apex and the two jet arms, which becomes unreliable at $z \lesssim z_{\text {col }}=32 \mu$ as due to instrumental resolution limitations (see text). The transition region is indicated with a vertical blue dashed line. A base-10 logarithmic scale is used for the horizontal axis.

manifests itself as interaction region between an accretion-powered outflow $^{15}$ and the fast jet spine, which is potentially powered by the black hole $\operatorname{spin}^{16}$. The mass-loaded sheath has a higher intrinsic emissivity compared with the evacuated spine. The same type of LLAGN-applicable GRMHD simulations also self-consistently develop a collimating helical magnetic field structure in the jet, which is confirmed observationally in many $\mathrm{AGN}^{17}$. The dominating sheath emissivity and helical magnetic field structure provides a natural intrinsic explanation for the prevailing edge-brightening in LLAGN and can also explain the northwest-southeast brightness asymmetry. This model and alternative geometric explanations for the brightness asymmetries are discussed in 'Brightness asymmetries' in Methods.

The basic radiative properties of these jets can be analytically understood with a simple model ${ }^{18}$, where particle and magnetic energy density equipartition is assumed, while the particle density decays with $z^{-2}$. Under these conditions, an optically thick and self-absorbed compact feature is expected (the core), whose position $z_{\text {core }}$ along the jet is frequency dependent with $z_{\text {core }} \propto \nu^{-1}$ (refs. ${ }^{1,20}$ ). This radio core corresponds to the photosphere, where the optical depth $\tau(\nu)$ to photons at the observing frequency $\nu$ is unity. The jet is optically thick upstream and optically thin downstream. The photosphere moves closer to the jet apex at higher frequencies, until the point where either the launching point is reached near the horizon, or particle acceleration has not yet begun ${ }^{21}$. The scale of a jet 'nozzle' emission cannot be smaller than the $\sqrt{27} r_{\mathrm{g}}$ photon capture radius ('The location of the black hole' in Methods).

The combination of all emission regions along the jet gives rise to a flat to inverted radio spectrum, peaking at a maximum frequency $\widetilde{\nu}$, determined by the black hole mass $M$ and accretion rate $\dot{M}$ and scaling as $\widetilde{\nu} \propto M^{-1} \dot{M}^{2 / 3} \propto M^{-1} F_{\mathrm{r}}^{8 / 17} D^{16 / 17}$ (refs. ${ }^{6,19,22}$ ). Here, $D$ is the distance of the black hole to the observer and $F_{\mathrm{r}}$ the observed radio flux density. These scaling relations follow from the assumption that the jet's internal gas and magnetic pressures are linearly coupled to the accretion rate and maintain a fixed ratio along the jets. The proportionality constant between $\dot{M}$ and $F_{\mathrm{r}}$ generally depends on the jet's velocity, electron and magnetic energy densities, particle distribution spectrum and inclination angle. Therefore, we are only able to make a first-order estimate. It should further be noted that X-ray binary observations ${ }^{23}$ have revealed a more complex relationship between $\widetilde{\nu}$ and $\dot{M}$, where the innermost particle acceleration zone in the jet may not remain stationary and source-specific accretion disk parameters come into play. The same effects are expected to also influence $\widetilde{\nu}$ in AGN, which substantiates the fact that only order-of-magnitude estimates can be provided for $\widetilde{\nu}$. We assume the brightest features in our image to correspond to the radio cores at $230 \mathrm{GHz}$, which is discussed in 'Alternative interpretations for the brightest jet features' in Methods. Our assumption is affirmed by three consistent and independent measurements of $\widetilde{\nu}$, but future 
spectral information is needed for a definitive confirmation. We show that $\widetilde{\nu}$ lies in the terahertz regime for Cen A based on the core shift that we can determine from our image, scaling relations with the M87 jet, and the spectral energy distribution of Cen A.

We take the distance from the brightest pixel in the image to the estimated position of the jet apex and obtain a core shift of $z_{\text {core }}=32 \pm 11 \mu$ as. On the basis of this distance and the uncertain inclination angle, we estimate that an observing frequency of $\widetilde{\nu}_{\text {CenA }} \approx 10-60 \mathrm{THz}$ (The location of the black hole' in Methods) will reach the base of the jet at the black hole innermost stable circular orbit. A caveat is that we do not take the effect of the uncertain ambient medium into account in this simple picture.

Independently, we can use the above scaling relations to estimate the order of magnitude of $\widetilde{\nu}_{\text {CenA }}$ by comparing the Cen A jet with M87, which has $\widetilde{\nu}_{\mathrm{M} 87}=228 \mathrm{GHz}$ (refs. ${ }^{24,25}$ ). For the centimetre jet radio core, a flux density of $\sim 1 \mathrm{Jy}$ is measured for both sources ${ }^{3,26}$, which yields $\dot{M}_{\mathrm{CenA}} \approx 0.1 \dot{M}_{\mathrm{M} 87}$ for the accretion onto the black hole and therefore $\widetilde{\nu}_{\mathrm{CenA}} \approx 26 \widetilde{\nu}_{\mathrm{M} 87} \approx 6 \mathrm{THz}$ ('The location of the black hole' in Methods), in agreement with our observations and the assumed position of the black hole at the jet apex within an order of magnitude. On the basis of comparable jet velocities $(\sim 0.3 c-0.5 c$, where $c$ is the speed of light) and inclination angles $\left(\sim 20^{\circ}\right)$, we have assumed the amount of Doppler boosting to be similar in both jets. The relation of accretion rates would constrain $\dot{M}_{\text {CenA }}$ to be $\lesssim 9 \times 10^{-5} M_{\odot} \mathrm{yr}^{-1}$ (ref. ${ }^{27}$ ) or $7 \times 10^{-5} \dot{M}_{\mathrm{Edd}}$ in terms of the Eddington accretion rate for an assumed radiative efficiency of $10 \%$.

The core spectral energy distribution of Cen A peaks at $\sim 10^{13} \mathrm{~Hz}$ (ref. ${ }^{28}$ ), which may be the equivalent of the submillimetre bump seen in Sagittarius $\mathrm{A}^{\star 29,30}$, and would further support our hypothesis.

Observed correlations between the masses of accreting black holes and their X-ray and radio luminosities form the basis of a unified fundamental plane of scale-invariant black hole accretion. This scale invariance has been derived based on stellar-mass black holes, which have a break frequency $\widetilde{\nu}_{\text {XRB }}$ in the near-infrared, and supermassive $10^{8} M_{\odot}-10^{10} M_{\odot} \mathrm{AGN}$, where the supermassive black hole $(\mathrm{SMBH})$ break frequency $\widetilde{\nu}_{\mathrm{SMBH}}$ lies in the radio to submillimetre regime ${ }^{5,6}$. With our observation, we demonstrate that the simple fundamental relations for the black hole jet activity still holds for a source with a mass of $5.5 \times 10^{7} M_{\odot}$ and $\widetilde{\nu}_{\mathrm{CenA}}$ in the terahertz regime, in between those two types of black hole. Our method used to determine the optimal frequency to observe black hole shadows based on core shift, jet power and source spectrum is in principle applicable to any LLAGN.

Our findings suggest that the black hole shadow ${ }^{4}$ of Cen A would be visible in a bright, optically thin accretion flow at an observing frequency of a few terahertz. At this high frequency, a VLBI experiment above Earth's troposphere would be able to resolve the $1.4 \pm 0.8 \mu$ as shadow diameter with a minimal baseline length of $\sim 8,000 \mathrm{~km}$.

\section{Methods}

Processing of observational data. This section describes the 2017 EHT observations of Cen A, the model-independent calibration ${ }^{9}$ with two separate pipelines, the flux density calibration, and known measurement issues and systematics with corresponding mitigation strategies. The final datasets coming out from the two pipelines are both used for the scientific analysis as cross-verification (Supplementary Fig. 2)

Data acquisition. Cen A (PKS 1322-428, hosted in the NGC5128 elliptical galaxy, $\left.\alpha_{\mathrm{I} 2000}=13 \mathrm{~h} 25 \min 27.62 \mathrm{~s}, \delta_{\mathrm{I} 2000}=-43^{\circ} 1^{\prime} 8.81^{\prime \prime}\right)$ was observed by the EHT in a six-hour-long track on 10 April 2017, with a total on-source integration time of 105 min (Supplementary Fig. 3). The observations were carried out by the Atacama Large Millimeter/submillimeter Array (ALMA), Atacama Pathfinder Experiment (APEX), James Clerk Maxwell Telescope (JCMT), Large Millimeter Telescope Alfonso Serrano (LMT), South Pole Telescope (SPT) ${ }^{31}$, Submillimeter Array (SMA) and Submillimeter Telescope (SMT) ${ }^{32}$. For ALMA, 37 of the $12 \mathrm{~m}$ dishes were phased-up ${ }^{33}$. From the Institut de Radioastronomie Millimétrique (IRAM), the IRAM $30 \mathrm{~m}$ telescope participating in the EHT observations is not able to see Cen A jointly with the rest of the array due to the low declination of the source. The data were recorded on Mark 6 VLBI recorders ${ }^{34}$ with 2-bit sampling in two $2 \mathrm{GHz}$ wide bands, 'low' and 'high', centred around $227.1 \mathrm{GHz}$ and $229.1 \mathrm{GHz}$, respectively. Unless stated otherwise, results are derived using the combined low-band + high-band data. Quarter-wave plates at each site except ALMA were used to observe circularly polarized light. The data were correlated with the Distributed FX (DiFX) software ${ }^{35,36}$. The PolConvert ${ }^{37}$ software was used to convert the phased ALMA data $^{33}$ from a linear polarization basis to a circular basis after correlation, based on solutions from the calibration of the connected-element ALMA data ${ }^{38}$.

Data reduction pipelines. The autocorrelation normalization, feed angle rotation, fringe fitting, bandpass calibration and a priori correction of atmospheric phase turbulence ${ }^{39}$ were performed independently by two pipelines: rPICARD $^{9}$, which is based on the Common Astronomy Software Applications (CASA) package ${ }^{40}$, and EHT-HOPS $^{10}$, which is based on the Haystack Observatory Postprocessing System $(\text { HOPS })^{41}$. DiFX produces Flexible Image Transport System Interferometry Data Interchange Convention (FITS-IDI) and Mark4 data. rPICARD uses the FITS-IDI product and converts it into the measurement set format. EHT-HOPS uses the Mark4 data. Both software packages convert the calibrated data into the UVFITS format for further processing.

rPICARD performs an upstream correction for the feed rotation angle and uses station-based global fringe fitting based on an unpolarized point source model to correct for phases, delays and rates consistently for the right-circular-polarization and left-circular-polarization signal paths ${ }^{42}$. Atmospheric phase and residual delay variations are corrected within the expected coherence time by fringe fitting segmented data of each VLBI scan. The segmentation length is set by the signal-to-noise ratio (SNR) of each baseline.

For EHT-HOPS, the feed rotation angle is corrected after the fringe fitting together with an additional polarization calibration step, where complex polarization gain offsets are solved for. Delays and rates are found in a baseline-based fringe search and referenced to individual stations with a least-squares optimization ${ }^{43}$. Atmospheric phases are corrected by fitting a polynomial phase model to the data on baselines to the most sensitive reference station in each scan. A round-robin approach is used to avoid fitting to thermal noise and the degree of the polynomial is set by the SNR of the data.

Gain amplitude calibration. The flux density calibration is done based on determined station sensitivities in a common framework for the rPICARD and EHT-HOPS data ${ }^{44}$. The sensitivity of a station $i$ is given by its system equivalent flux density $\left(\mathrm{SEFD}_{i}\right)$ in Jy, which takes into account the gain and total noise power along a telescope's signal chain as a function of time $t$ and frequency $\nu$. On a baseline $i-j$, correlation coefficients $\xi_{(i, j)}$ in units of thermal noise are calibrated to a physical radiation intensity scale of correlated flux density $\mathcal{S}_{(i, j)}$ through

$$
\mathcal{S}_{i, j}(t, \nu)=\frac{\sqrt{\operatorname{SEFD}_{i}(t, \nu) \operatorname{SEFD}_{j}(t, \nu)}}{\eta_{\mathrm{Q}}} \xi_{i, j}(t, \nu)
$$

where $\eta_{\mathrm{Q}}$ is the quantization efficiency. For data recorded with 2-bit sampling, we have $\eta_{\mathrm{Q}} \approx 0.88$.

The gains of co-located stations were solved based on a contemporaneous measurement of the total flux density $S_{0}=5.62 \mathrm{Jy}$ of the source with the ALMA interferometer ${ }^{10,38,44}$. The correlated flux $\mathcal{S}$ measured between two co-located sites $p$ and $q$ should be equal to $S_{0}$ and for a third station $o$, we should have $\mathcal{S}_{p o}=\mathcal{S}_{q o}$. It follows, that we can solve for the station-based amplitude gains $\mathcal{A}$ of $p$ and $q$ with a self-calibration approach. Here the model is given by the constant flux density $S_{0}$ seen by baselines between co-located sites. No gain corrections for non-co-located ('isolated') stations are solved for.

Ad hoc correction factors are used to correct signal losses at APEX due to an injected instrumental signal and at SMA due to temporary losses of bandwidth ${ }^{44}$ In addition, LMT and SPT suffered from pointing problems, which result in substantial amplitude variations between and within VLBI scans. These losses cannot be estimated a priori and must be corrected with self-calibration gain solutions derived within short $\sim 10$ s segments from high SNR data. The SMT station was able to track the source down to an elevation of a few degrees. Large self-calibration gain factors are therefore needed towards the end of the experiment. Besides these known data issues, gain corrections factors are well constrained within a determined a priori error budget ranging between $10 \%$ and $20 \%$ for the individual stations ${ }^{44}$

Imaging. In this section, we describe how we obtained our image model from the observational data. In a first step, we have established a blind consensus between different imaging methods. Then, we have fine-tuned the parameters of one method, eht-imaging ${ }^{45,46}$, for the rPICARD and EHT-HOPS data to obtain final images for the analysis of the Cen A jet structure.

The highest-resolution images of this southern source before this work were obtained within the Tracking Active Galactic Nuclei with Austral Milliarcsecond Interferometry (TANAMI) program ${ }^{47}$ at $8 \mathrm{GHz}$ and $22 \mathrm{GHz}$ with a maximum resolution of $400 \mu \mathrm{as}$, showing an extremely collimated structure with multiple distinct radio knot emission regions ${ }^{3}$. In a previous single-baseline non-imaging study of Cen A, a bright compact core was detected at $215 \mathrm{GHz}$ (ref. ${ }^{48}$ ). 
Blind challenge. Similarly to the method used when the shadow of M87* was resolved by the $\mathrm{EHT}^{25,49}$, we have carried out a blind imaging challenge before proceeding to the scientific analysis of the data. In this challenge, a number of individuals have reconstructed an image of the source independently of each other. Early (not fully verified) low-band data from the EHT-HOPS pipeline was used, which had slightly larger amplitude gain errors from outdated a priori calibration parameters. Out of twelve total images, six had acceptable reduced $\chi_{\mathrm{cp}}^{2}<2$ for the closure phases. These images were obtained with the eht-imaging and SMILI ${ }^{50,51}$ regularized maximum likelihood methods and the Difmap ${ }^{52,53}$ and CASA ${ }^{9,54}$ CLEAN methods ${ }^{55,56}$. The images that did not make the $\chi^{2}$ cut often showed spurious emission features and strong sidelobe structures.

Final imaging method. With the imaging challenge, we have established that different methods converge towards the same robust source structure (Supplementary Fig. 2), independent of shared human bias. Further imaging analysis of the rPICARD and EHT-HOPS science release data was pursued with the final M87* eht-imaging script ${ }^{49}$, which is based on application of a regularized maximum likelihood method that includes a maximum entropy term. Using a second-moment-based pre-calibration, LMT gains were stabilized with respect to the better constrained SMT amplitudes ${ }^{57}$. As Cen A is sufficiently compact within the EHT beam, the short LMT-SMT baseline measures a Gaussian-like source structure. We have performed an initial self-calibration to a Gaussian with size $\Theta_{\text {maj }} \times \Theta_{\text {min }}$ at a position angle $\Theta_{\mathrm{PA}}$ and with a total flux of $S_{\mathrm{G}}$. Here, $\Theta_{\text {maj }}$ and $\Theta_{\text {min }}$ are the major and minor axis sizes of the Gaussian in radians. Any gains that were erroneously introduced in this process can later be reconciled in image-based self-calibration steps. To solve for the image brightness distribution $\mathcal{I}$ with a regularized maximum likelihood method (employed by eht-imaging), we are minimizing

$$
\sum_{D} \alpha_{D} \chi_{D}^{2}(\mathcal{I})-\sum_{R} \beta_{R} \Lambda_{R}(\mathcal{I})
$$

Here $D$ represents the collection of data terms, which are derived from the measured visibilities and have approximately normal noise statistics ${ }^{58}$ : amplitudes, closure phases and log closure amplitudes. Corresponding to each data term, we have a goodness-of-fit function $\chi_{D}^{2}=\left\{\chi_{\mathrm{amp}}^{2}, \chi_{\mathrm{cp}}^{2}, \chi_{\mathrm{lca}}^{2}\right\}$ and relative weighting $\alpha_{D}=\left\{\alpha_{\mathrm{amp}}, \alpha_{\mathrm{cp}}, \alpha_{\mathrm{lca}}\right\}$, We have performed four incremental imaging runs with subsequent self-calibration, over which we have increased the weight of each data term: $\alpha_{D}^{(1)} \rightarrow \alpha_{D}^{(2)} \rightarrow \alpha_{D}^{(3)}$ with $\alpha_{D}^{(1)}<\alpha_{D}^{(2)}<\alpha_{D}^{(3)} \forall D$. Regularizer terms $\Lambda_{R}$ are included with weights $\beta_{R}$ to impose additional assumptions on the image. We have imposed two regularization parameters: one for a maximum entropy method $(\mathrm{MEM})^{59}$ term with weight $\beta_{\mathrm{MEM}}$ and another one for the amount of compact flux $Z_{0}$ in the image with weight $\beta_{z}$. The MEM term minimizes the entropy of $\mathcal{I}$ with respect to a prior image $\Phi$, which results in a similarity between the two images for each pixel $i$. Here, we used $\Lambda_{\text {MEM }}=-S_{0}^{-1} \sum_{\mathrm{i}} \mathcal{I}_{i} \log \left(\mathcal{I}_{i} / \Phi_{i}\right)$. For the MEM prior image $\Phi$, we have chosen a Gaussian model oriented along the direction of the large-scale jet, which we also used as initialization for our imaging. It is expected that $Z_{0}<S_{0}$, as a substantial portion of the flux measured by ALMA may come from different emission mechanisms and larger scales outside of the EHT field of view. In fact, the $\sim 150 \mathrm{~m}$ JCMT-SMA baseline sees a flux density of about $5 \mathrm{Jy}$ and at $2 \mathrm{~km}$, ALMA-APEX recovers only $\sim 4 \mathrm{Jy}$. For M87* the EHT measured $Z_{0} \approx S_{0} / 2$ (ref. $^{49}$ ).

The numerical values of the final imaging parameters are given in Supplementary Table 1. Optimal parameters were chosen based on an empirical minimization of $\chi_{D}^{2}$, median station gains $\mathcal{A}^{(\mathrm{sc})}$ from self-calibration and patches of spurious flux in the image. In addition, we took the similarity of image reconstructions from the rPICARD and EHT-HOPS data for a given set of parameters into account to avoid overfitting to data peculiarities that result from assumptions made during the data calibration. A variety of images that can be reconstructed with various combinations of the free imaging parameters can be shared upon reasonable request. We have chosen for an eht-imaging reconstruction of the rPICARD data for our final image, as this imaging method and dataset have been studied most extensively.

Our images are shown in units of brightness temperature $\mathcal{T}(\mathrm{K})$, which is related to a flux density $S$ in Jy through the observing wavelength $\lambda$, Boltzmann constant $k_{\mathrm{B}}$ and angular resolution element $\Omega$ as $\mathcal{T}=\lambda^{2}\left(2 k_{\mathrm{B}} \Omega\right)^{-1} S$.

Fundamental data properties and fits of the final image model to the data are shown in Supplementary Fig. 4. In Supplementary Fig. 5, we show the measured amplitudes projected along and perpendicular to the jet position angle. Along the jet axis, amplitudes fall off quickly at long projected baseline lengths, indicating the absence of substructures along the jet. Perpendicular to the jet, 'bouncing' amplitudes out to large projected baseline lengths occur, due to the strong intensity gradients across the transverse jet profile.

Synthetic data imaging tests. We have used the SYMBA software ${ }^{60}$ to perform imaging studies based on simulated observations. Given an input source model $\mathcal{M}$, SYMBA follows the entire EHT signal path to predict which source structure $\mathcal{I}$ would eventually be reconstructed. Thereby, we can assess how close our image reconstruction comes to the ground truth structure $\mathcal{M}$ of a fabricated observed source. SYMBA simulates the parameters of Earth's atmosphere with the
ATM module ${ }^{61}$ to add sky noise, signal attenuation and phase turbulence. Next, gain, leakage, pointing and focus errors plus thermal noise are introduced for each telescope in the array based on known telescope properties ${ }^{62}$. Afterwards, the simulated corrupted data are calibrated by rPICARD in the same way as observational data. The $u, v$ coverage and SEFD sensitivities are taken from the 2017 Cen A EHT observation track. The simulated calibrated data are then imaged with the same final eht-imaging script used to image the observational data in this work.

To assess the robustness of secondary features in our image reconstruction, we have performed three synthetic data tests (Supplementary Fig. 1). First, a control study to demonstrate that the output reconstruction from SYMBA correctly matches the input model $\mathcal{M}_{\text {final }}$. Then, we have removed the counterjet and emission features at large distance to the apex $z$ from $\mathcal{M}_{\text {final }}$ to verify that these do not spuriously appear in our simulated observation.

Furthermore, we have explored the upper limit on the brightness $\mathcal{T}_{\text {sp }}$ of potential emission from the jet spine by adding a weak emission component in the central jet region to $\mathcal{M}_{\text {final }}$. The goal was to find the smallest $\mathcal{T}_{\text {sp }}$, which would still be registered as an emission region in the reconstructed image $\mathcal{I}\left(\mathcal{M}_{\text {final }}+\mathcal{T}_{\text {sp }}\right)$.

Jet structure analysis. This section describes how we extract fundamental jet parameters from our image based on geometric arguments.

The position of the jet apex. We can empirically determine the approximate position of the jet apex, where the jet and counterjet are being launched, from the high-resolution image model shown in Fig. 2. A zoomed-in version of this plot is shown in Supplementary Fig. 6, which is overlaid with visual aids for the determination of the apex location. The first consideration is that the apex should be located in the region where the streamlines of the approaching jet converge. In our image, this convergence region lies upstream of the optically thick radio cores (assumed to correspond to the brightest regions of the jet) for both the northwest and southeast jet arms. Here we are limited by the resolution of our instrument, but a tentative merge of the two arms can be seen. The upper arm (region I in the figure) exhibits a strong bend, while the lower arm (IV) remains mostly straight. We note that a similar structure, where one jet arm appears to be straighter than the other one, is also present in the M87 jet ${ }^{26}$. The second consideration is the symmetry between the approaching jet and the counterjet. We note that there is no clear correspondence between individual features in the jet and counterjet. The counterjet appears straight with two components in the upper region (II) and one component in the lower region (III). As the apex must be upstream of the counterjet, the closest component of the receding jet to the approaching jet constrains how far upstream of the approaching jet the apex position can be. In fact, the position we assume for the apex based on the first consideration, where the streamlines of the approaching jet converge, lies halfway between the radio core in region I and the closest counterjet component in region II. It should be noted that a simple extrapolation of only the edge-brightened approaching jet would place the apex well inside the faint counterjet region.

On the basis of the robustness of our image reconstructions with different datasets, software packages and imaging parameters, we assume a positional uncertainty of $5 \mu$ as for the robust features of the image model, which is in agreement with the width of the jet ridgelines. Taking all constraints on the apex location into account, we estimate an uncertainty of $10 \mu$ as on the position.

For the determination of $z_{\text {core }}$, the pixel and jet apex position uncertainties are added in quadrature. On the basis of possible jet apex positions within the estimated uncertainty, we fit the $W \propto z^{k}$ jet profile multiple times and derive a systematic error of \pm 0.06 on $k$. When we used image model convolved with the nominal resolving beam, we obtain $k=0.35$ with a statistical error of \pm 0.2 .

Brightness asymmetries. The jet-counterjet asymmetry is most likely caused by relativistic boosting. We can calculate the $\mathcal{R}_{\mathrm{j} / \mathrm{cj}}$ brightness ratio by taking the average image flux density within $50 \times 100 \mu$ as rectangular regions on opposite sides of the apex. This ratio has to be interpreted with care, since the two regions may be at different distances to the jet apex. Moreover, counterjet radiation may be absorbed by the accretion flow and intrinsic jet-counterjet differences may arise from asymmetries in the jet launching process and the ambient medium ${ }^{63,64}$.

If we assume the intrinsic emissivity to be the same in the jet sheath and spine, beaming effects can be invoked to explain observed differences in brightness across the jet. We note that the intrinsic emissivity of the jet sheath is probably larger than that of the spine, as mentioned in the main text. The simplifying assumption of identical intrinsic emissivities can nonetheless be used to derive straightforward estimates for jet velocity components and the inclination angle $\theta$, since Doppler boosting is expected to have a considerable contribution to the observed source structure. If the inclination angle $\theta$ is not too small, a substantial portion of the spine emission may be beamed away from the line of sight. If the sheath and spine velocities are $c \beta_{\mathrm{sh}}$ and $c \beta_{\mathrm{sp}}$, respectively, the ratio of $I_{\mathrm{sh}}$ sheath and $I_{\mathrm{sp}}$ spine intensities in a continuous jet follows as

$$
\mathcal{R}_{\mathrm{sh} / \mathrm{sp}} \equiv \frac{I_{\mathrm{sh}}}{I_{\mathrm{sp}}}=\left[\frac{\sqrt{1-\beta_{\mathrm{sh}}^{2}}\left(1-\beta_{\mathrm{sp}} \cos (\theta)\right)}{\sqrt{1-\beta_{\mathrm{sp}}^{2}}\left(1-\beta_{\mathrm{sh}} \cos (\theta)\right)}\right]^{2-\alpha},
$$


with $\alpha\left(I \propto \nu^{\alpha}\right)$ as the spectral index of the optically thin jet components. Assuming a typical spectral index of $\alpha=-0.7$ and identical intrinsic emissivities, we can constrain the sheath and spine velocities with equation (3) and $\mathcal{R}_{\text {sh/sp }}>5$ to

$$
\left(1-\beta_{\mathrm{sp}}^{2}\right)^{-0.5}\left(1-\beta_{\mathrm{sp}} \cos (\theta)\right) \gtrsim 1.8\left(1-\beta_{\mathrm{sh}}^{2}\right)^{-0.5}\left(1-\beta_{\mathrm{sh}} \cos (\theta)\right) .
$$

$\left(1-\beta^{2}\right)^{-0.5}(1-\beta \cos (\theta))$ has a minimum of $\sqrt{1-\cos ^{2}(\theta)}$ at $\beta=\cos (\theta)$. It follows that the sheath-spine asymmetry can only be explained via beaming for $\beta_{\mathrm{sp}}>\cos (\theta)$, independent of the assumed value for the spectral index.

For Cen A, the jet spine emission may be beamed away from the line of sight, when its velocity exceeds $0.7 c-0.9 c$, while the sheath moves with a slower velocity. In fact, the emitting plasma of the large-scale jet was observed to move with $0.24 c-0.37 c$ (ref. $\left.^{3}\right)$.

For a full three-dimensional picture of a jet, where we assume the sheath to be symmetric in the $\phi$ direction around the spine in a cylindrical coordinate system, different spine and sheath emissivities, due to beaming or intrinsic effects, cannot, on their own, explain edge-brightening. The reason is that the sheath emission will contribute to any sightline towards the jet. A more detailed description, where also the optical depth is taken into account, is given in the next paragraph. In the remainder of this section, we go through the different scenarios that could cause the observed edge-brightening. First, we discuss a common interpretation related to pathlength differences. As this only works in optically thin regions, we put the presence of helical magnetic fields forward as the most likely, intrinsic explanation for edge-brightening in LLAGN. We then discuss more exotic scenarios, of a rotating or asymmetric jet, which might be tested through future observations.

In the optically thin jet regions, the integrated column density along sightlines through the jet at different distances from its axis (centre versus edges) can be used to explain edge-brightening. These are sightlines that, across the transverse extent of the jet, enter the jet at different locations. The sightlines first pass through the near side of the jet and exit again at the other side of the jet, the far side. If we assume the absence of intrinsic spine emissivity (due to weak mass loading or beaming of radiation into a narrow cone away from the line of sight), the observed radiation will be produced by a sheath of thickness $\Delta R$. For a line sight that goes exactly through the centre of the jet, we pass twice through the sheath, which would amount to a pathlength of $2 \Delta R / \sin \theta$, when the pathlength is short enough to locally approximate the jet as a cylinder. For a local jet radius $R_{\mathrm{i}}$, the column density along a sightline through the edge of the jet will be larger by a factor of $\sim \sqrt{R_{\mathrm{j}} / \Delta R}$ (ref. ${ }^{26}$ ). Here we have neglected changes in emissivity as sightlines pass through material at different distances to the jet apex. This simple model is capable of explaining edge-brightening in optically thin jet regions, where radiation along longer pathlengths accumulates. For Cen A, this would imply a thin radiating sheath with $\Delta R<0.04 R_{\text {. }}$

However, the edge-brightening in Cen A extends to the presumably optically thick radio core, suggesting that different physics are at play in this jet. The likely presence of a helical magnetic field ${ }^{17,65-67}$ combined with a rotating sheath and the inclination angle $\theta$, can lead to favourable/unfavourable pitch angles that maximize/minimize the synchrotron emissivity along the edges/centre of the jet. For a power-law distribution of electrons with index $p$, where in the rest frame of the jet, the electron density $n$ follows their energy $E$ as $\mathrm{d} n \propto E^{-p} \mathrm{~d} E$, the synchrotron emission coefficient in the rest frame scales as $j_{\nu} \propto|B \sin \chi|^{(p+1) / 2} \nu^{-(p-1) / 2}$ (refs $\left.{ }^{68,69}\right)$. Here, $B$ is the magnetic field strength, $\chi$ is the angle between the magnetic field and line of sight, and $\nu$ is the radiation frequency. The corresponding absorption coefficients scale as $\alpha_{\nu} \propto|B \sin \chi|^{(p+2) / 2} \nu^{-(p+4) / 2}$ (refs. $\left.{ }^{68,69}\right)$. It can be seen that no asymmetries in $\chi$ would arise across the transverse jet profile for a purely poloidal $\left(B_{z}\right)$ magnetic field. The edge-brightening is maximized for perpendicular angles $\chi$ between the line of sight and magnetic field at the jet edges, while the magnetic field is oriented parallel to the line of sight in the centre of the jet. In future work, we will study the polarimetric properties of the jet with the EHT to test this hypothesis as explanation for the edge-brightening. To get a handle on $\chi$, it will be necessary to narrow down the inclination angle $\theta$ and jet velocity with monitoring observations to take relativistic aberration into account.

For optically thick jet regions upstream of the radio core, the relativistic boosting is sensitive to the shape of the emitting region and less sensitive to the Doppler factor ${ }^{18}$. In the presence of a fast helical jet flow and $\theta>0$, part of the jet will rotate towards the observer and the other part will rotate in the opposite direction on the sky. Beyond the initial jet launching region, the jet is strongly collimated and the viewing angle to the jet edges will be very close to $\theta$. For a flow with toroidal and poloidal components, we denote the angle of the helical velocity component $\beta_{\mathrm{h}}$ with respect to the poloidal direction along the line of sight with $\phi_{\mathrm{h}}$. For two identically shaped, optically thick radio core components of intensity $I_{\mathrm{s}}$ at the southeast jet edge and $I_{\mathrm{n}}$ at the northwest edge, we thus have ${ }^{26}$

$$
\mathcal{R}_{\mathrm{s} / \mathrm{n}} \equiv \frac{I_{\mathrm{s}}}{I_{\mathrm{n}}}=\left[\frac{1-\beta_{\mathrm{h}} \cos \left(\theta+\phi_{\mathrm{h}}\right)}{1-\beta_{\mathrm{h}} \cos \left(\theta-\phi_{\mathrm{h}}\right)}\right]^{2}
$$

For an anticlockwise jet rotation and $\mathcal{R}_{\mathrm{s} / \mathrm{n}} \approx 1.6$, we get the weak constraint of

$$
1.3 \cos \left(\theta-\phi_{\mathrm{h}}\right)-\cos \left(\theta+\phi_{\mathrm{h}}\right) \simeq 0.3 \beta_{\mathrm{h}}^{-1}
$$

When the bulk velocities of the northwest and southeast jet sheaths are known, $\phi_{\mathrm{h}}$ and subsequently $\beta_{\mathrm{h}}$ can be determined ${ }^{26}$. We note that the the small linear scales resolved by the EHT in Cen A uniquely allow us to track relativistic dynamics across days in this source with future observations.

In an alternative scenario, this tentative northwest-southeast brightness asymmetry seen in Cen A could be explained with two distinct jet components having different velocities or different inclinations angles with respect to the line of sight.

In this work, we have interpreted the edge-brightening in terms of a naturally emerging spine-sheath jet structure in LLAGN, based on results from GRMHD simulations that are applicable to those type of sources. However, the same phenomenon is also observed in more powerful AGN; for example, Cygnus $\mathrm{A}^{70,71}$, where an accretion flow operating at $\sim 1 \%$ of the Eddington limit is unlikely to be radiatively inefficient ${ }^{72}$

Collimation profile. Following the northwest and southeast jet ridgelines, we bin distance values to the jet apex into intervals of $10 \mu$ as in size. Within each bin, we select the brightest pixel to obtain the central location along the ridge. We impose a statistical uncertainty of $5 \mu$ as on distances $z$ in accordance with the width of the jet ridgelines in our image model. The width $W$ of the jet is taken as the distance between the two jet arms. The profile of our image is shown in Fig. 3 together with the corresponding average opening angle computed from the jet width as a function of distance to the apex.

Resolution limitations prevent us from tracing down the exact value of the initial jet opening angle $\psi_{\text {iet }}$ near the apex, where the analysis of binned distance values becomes uncertain. Nonetheless, we can derive an upper limit on $\psi_{\text {jet }}$ with a simple geometric argument: the jet has a clearly defined collimation region beyond

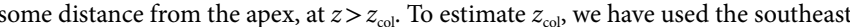
jet arm, as it is brighter, straighter, and has a more clearly identifiable compact brightness core. If we now assume that the jetstream converges monotonically towards the apex for $z<z_{\text {col }}$ and that the apex itself does not correspond to an extended region, we have

$$
\psi_{\text {jet }} \geq 2 \arctan \left(\frac{W\left(z_{\mathrm{col}}\right)}{2 z_{\mathrm{col}}}\right)
$$

If the inclination angle $\theta$ is known, the intrinsic opening angle $\psi_{\text {int }}$ can be computed as ${ }^{73}$

$$
\psi_{\text {int }} \geq 2 \arctan \left(\sin (\theta) \frac{W\left(z_{\text {col }}\right)}{2 z_{\text {col }}}\right)=2 \arctan \left(\sin (\theta) \tan \left(\frac{\psi_{\text {jet }}}{2}\right)\right) .
$$

The jet remains collimated out to kiloparsec scales and contains multiple particle acceleration sites in a knotted structure ${ }^{3,74-76}$. The source is a well-suited laboratory for models of AGN feedback ${ }^{77,78}$ and the creation of ultrahigh-energy cosmic rays ${ }^{79,80}$.

Confinement by the ambient medium. Analytic theory for axisymmetric, relativistic Poynting-dominated outflows can be used to derive exact asymptotic solutions for the influence an ambient medium on the collimation of a jet. One can show that in the presence of external pressure gradient $P_{\text {ext }}(z)=P_{0} z^{-\kappa}$, the jet expansion profile $W$ as a function of distance along the jet axis $z$ follows ${ }^{81,82}$

$$
\frac{\mathrm{d}^{2} W}{\mathrm{~d} z^{2}}-W^{-3}+C_{1} P_{0} z^{-\kappa} W=0
$$

in a simplified form, with $C_{1}$ a numerical constant. At large $z$ and for a shallow external pressure gradient with $\kappa<2$, we obtain ${ }^{81,82}$

$$
W(z)=C_{1}^{-1 / 4} \sqrt{\frac{2-\kappa}{\pi}} \sqrt{C_{2}^{-1} \cos ^{2} S+C_{2}\left(C_{3} \cos S+\frac{\pi}{2-\kappa} \sin S\right)} z^{\kappa / 4},
$$

for $S(z)=C_{4} z^{1-\kappa / 2}-C_{5}$, and $C_{2}, C_{3}, C_{4}$ and $C_{5}$ numerical constants. Equation (10) shows that the ambient pressure will confine the jet into a $W \propto z^{k}$ profile with $k=\kappa / 4$. In addition, oscillations along the jet boundary can occur in a non-equilibrium state for $C_{2} \neq(2-\kappa) / \pi, C_{3} \neq 0$ (ref. ${ }^{82}$ ).

The location of the black hole. Given a measurement of the core shift $z_{\text {core }}$ with respect to the black hole, we can gauge the observing frequency $\widetilde{\nu}$, which corresponds to a small self-absorbed nozzle region at the footprint of the jet ${ }^{83}$. This region corresponds to a peak or break from a jet-dominated flat radio spectrum as it is the smallest region where particle acceleration can occur. The minimum scale where a jet can be launched by a black hole is given by the innermost stable circular orbit. The size of the emission region of this nozzle would be given by the photon capture radius. Thus, we can estimate $\widetilde{\nu}$ as

$\widetilde{\nu}=\frac{\nu_{\mathrm{obs}} z_{\mathrm{core}}\left(\nu_{\mathrm{obs}}\right)}{\sqrt{27} r_{\mathrm{g}}} \simeq 20\left(\frac{\nu_{\mathrm{obs}}}{\mathrm{GHz}}\right)\left(\frac{z_{\mathrm{core}}\left(\nu_{\mathrm{obs}}\right)}{\mu \mathrm{as}}\right)\left(\frac{D}{\mathrm{Mpc}}\right)\left(\frac{M}{10^{6} M_{\odot}}\right)^{-1}$ 
In this expression, $M$ is the mass of the black hole and $D$ the distance from the black hole to the observer.

With the derived scaling relation of $\widetilde{\nu} \propto M^{-1} \dot{M}^{2 / 3} \propto M^{-1} F_{\mathrm{r}}^{8 / 17} D^{16 / 17}$, we can relate the break frequencies of two sources if their accretion rates or jet properties are known. Here, $\dot{M}$ is the black hole accretion rate and $F_{\mathrm{r}}$ is the observed flat-spectrum radio flux density. In particular, if we assume for two sources to share the same basic intrinsic jet properties and orientation with respect to Earth, we have

$$
\frac{\widetilde{\nu_{1}}}{\widetilde{\nu_{2}}}=\left(\frac{F_{\mathrm{r} 1}}{F_{\mathrm{r} 2}}\right)^{8 / 17}\left(\frac{D_{1}}{D_{2}}\right)^{16 / 17}\left(\frac{M_{1}}{M_{2}}\right)^{-1} .
$$

While these expressions are strictly speaking only true for a filled conical jet, they appear to describe the emission from the jet sheath and its basic scaling properties reasonably well ${ }^{84-86}$ and allows one to make a first-order estimate of the characteristic radio frequency of near-horizon emission.

We have used the above equations to estimate the accretion rate of Cen A to the one of M87 based on the assumption of a similar coupling between $\mathrm{SMBH}$ inflows and jet power. External Faraday rotation effects and a generally variable rotation measure further complicates the assumed relation of accretion rates $^{87}$, which should thus be taken as only an order-of-magnitude estimate. It is, however, worth pointing out that the black hole growth rate measured over cosmic timescales from X-ray cavity fluxes from the jet radio lobes is $\sim 10^{-3} M_{\odot} \mathrm{yr}^{-1}$ for both Cen $\mathrm{A}$ and $\mathrm{M} 87^{88}$

Alternative interpretations for the brightest jet features. In this work, we have interpreted the brightest jet features as radio cores, which mark the transition region between upstream synchrotron self-absorbed jet regions and downstream optically thin areas. In our image, we are able to resolve the self-absorbed region between the putative radio core and jet apex, which coincides with the location of the SMBH and its accretion disk. With current telescopes, the radio core and upstream region remains unresolved for most AGN (see Table 2 in ref. ${ }^{1}$ for example).

The radio core interpretation of the brightest jet features seems most plausible given our data. On the basis of simple analytical jet theory, a bright radio core is expected to be present in VLBI images. Radio cores are typically seen in sources similar to Cen A and the core shift typically follows the standard $\nu^{-1}$ relation in most sources ${ }^{89}$. In fact, special circumstances have to be invoked to explain the absence of radio cores in VLBI images. For example, obscuration by an optically thick region in the foreground. We do think that this is a likely scenario for our observation given the small scales probed in our image, the high observing frequency and the proximity of the source. Moreover, the core shift we have computed in Cen A agrees with the core SED of the source and fundamental plane equations that relate the jet power of Cen A to the M87 jet power. Our image is dominated by the brightest, compact jet features, which would be weakly polarized and have flat spectrum as radio cores ${ }^{1}$. It should be noted that wide-bandwidth ALMA interferometer data, which were taken simultaneously with the VLBI observations, show a flat spectrum between $212 \mathrm{GHz}$ and $230 \mathrm{GHz}$ and place a $3 \sigma$ upper limit of $0.15 \%$ on the linear polarization fraction ${ }^{87}$. The ALMA measurements are, however, at a larger arcsecond resolution and we resolve out $64 \%$ of the flux measured by ALMA with the EHT. We therefore need future polarimetric and spectral VLBI results for confirmation.

However, with the current observations, we cannot conclusively rule out the possibility that there is insufficient particle acceleration in the jet, such that no radio core is formed at $\lambda 1.3 \mathrm{~mm}$, while a core is present at longer wavelengths ${ }^{3,76}$. In this scenario, the bright jet regions would most probably correspond to a shock within the jet flow. The strongest counterargument here is that the radio spectrum of the core turns over at terahertz frequencies. This emission is most likely produced by the jet, ergo, particle acceleration should occur up to the energies that produce terahertz synchrotron emission.

\section{Data availability}

The ALMA raw visibility data can be retrieved from the ALMA data portal under the project code 2016.1.01198.V. The calibrated Stokes I VLBI visibility data of Centaurus A can be obtained from a DOI listed under https:// eventhorizontelescope.org/for-astronomers/data with the code 2021-D0301. Image FITS files and scripts to reproduce the plots are available from the corresponding author upon reasonable request.

\section{Code availability}

Antenna gains that enter the SEFDs were computed with https://bitbucket.org/M Janssen/eht-flux-calibration. The SEFDs were applied with the https://github.com/ sao-eht/eat code, which also contains the EHT-HOPS pipeline. rPICARD is hosted on https://bitbucket.org/M_Janssen/picard. Configuration and run files, which make use of self-contained Docker images are at https://bitbucket.org/M_Janssen/ casaeht. This work is based on the 'ER6' data production scripts, for which the 30e6ca14fb50275013c668285a3b476f9bc85436_91da63236db34f3a31b5309b18ac1 $59128 \mathrm{f} 28 \mathrm{a} 35$ image was used.
The eht-imaging software is hosted on https://github.com/achael/eht-imaging. SYMBA is at https://bitbucket.org/M_Janssen/symba. The docker image used here is tagged as dec65699ccc0acdc6e6ba8f218d6724537fc613a and can be found on https://hub.docker.com/r/mjanssen2308/symba.

Received: 22 January 2021; Accepted: 2 June 2021; Published online: 19 July 2021

\section{References}

1. Boccardi, B., Krichbaum, T. P., Ros, E. \& Zensus, J. A. Radio observations of active galactic nuclei with mm-VLBI. Astron. Astrophys. Rev. 25, 4 (2017)

2. Harris, G. L. H., Rejkuba, M. \& Harris, W. E. The distance to NGC 5128 (Centaurus A). Publ. Astron. Soc. Aust. 27, 457-462 (2010).

3. Müller, C. et al. TANAMI monitoring of Centaurus A: the complex dynamics in the inner parsec of an extragalactic jet. Astron. Astrophys. 569, A115 (2014)

4. Falcke, H., Melia, F. \& Agol, E. Viewing the shadow of the black hole at the Galactic Center. Astrophys. J. Lett. 528, L13-L16 (2000).

5. Merloni, A., Heinz, S. \& diMatteo, T. A fundamental plane of black hole activity. Mon. Not. R. Astron. Soc. 345, 1057-1076 (2003).

6. Falcke, H., Körding, E. \& Markoff, S. A scheme to unify low-power accreting black holes. Jet-dominated accretion flows and the radio/X-ray correlation. Astron. Astrophys. 414, 895-903 (2004).

7. Neumayer, N. The supermassive black hole at the heart of Centaurus A revealed by the kinematics of gas and stars. Publ. Astron. Soc. Aust. 27, 449-456 (2010).

8. Chatterjee, K., Liska, M., Tchekhovskoy, A. \& Markoff, S. B. Accelerating AGN jets to parsec scales using general relativistic MHD simulations. Mon Not. R. Astron. Soc. 490, 2200-2218 (2019).

9. Janssen, M. et al. rPICARD: A CASA-based calibration pipeline for VLBI data. Calibration and imaging of $7 \mathrm{~mm}$ VLBA observations of the AGN jet in M 87. Astron. Astrophys. 626, A75 (2019).

10. Blackburn, L. et al. EHT-HOPS pipeline for millimeter VLBI data reduction. Astrophys. J. 882, 23 (2019).

11. Kim, J.-Y. et al. The limb-brightened jet of M87 down to the 7 Schwarzschild radii scale. Astron. Astrophys. 616, A188 (2018).

12. Piner, B. G., Pant, N., Edwards, P. G. \& Wiik, K. Significant limb-brightening in the inner parsec of Markarian 501. Astrophys. J. Lett. 690, L31-L34 (2009)

13. Giovannini, G. et al. A wide and collimated radio jet in $3 \mathrm{C} 84$ on the scale of a few hundred gravitational radii. Nat. Astron. 2, 472-477 (2018).

14. Narayan, R., Mahadevan, R. \& Quataert, E. in Theory of Black Hole Accretion Disks (eds Abramowicz, M. A. et al.) 148-182 (Cambridge Univ. Press, 1998).

15. Blandford, R. D. \& Payne, D. G. Hydromagnetic flows from accretion disks and the production of radio jets. Mon. Not. R. Astron. Soc. 199, 883-903 (1982)

16. Blandford, R. D. \& Znajek, R. L. Electromagnetic extraction of energy from Kerr black holes. Mon. Not. R. Astron. Soc. 179, 433-456 (1977).

17. Gabuzda, D. Evidence for helical magnetic fields associated with AGN jets and the action of a cosmic battery. Galaxies 7, 5 (2018).

18. Blandford, R. D. \& Königl, A. Relativistic jets as compact radio sources. Astrophys. J. 232, 34-48 (1979).

19. Falcke, H. \& Biermann, P. L. The jet-disk symbiosis. I. Radio to X-ray emission models for quasars. Astron. Astrophys. 293, 665-682 (1995).

20. Lobanov, A. P. Ultracompact jets in active galactic nuclei. Astron. Astrophys. 330, 79-89 (1998).

21. Romero, G. E., Boettcher, M., Markoff, S. \& Tavecchio, F. Relativistic jets in active galactic nuclei and microquasars. Space Sci. Rev. 207, 5-61 (2017).

22. Heinz, S. \& Sunyaev, R. A. The non-linear dependence of flux on black hole mass and accretion rate in core-dominated jets. Mon. Not. R. Astron. Soc. 343, L59-L64 (2003).

23. Lucchini, M. et al. Correlating spectral and timing properties in the evolving jet of the micro blazar MAXI J1836-194. Mon. Not. R. Astron. Soc. 501, 5910-5926 (2020).

24. Hada, K. et al. An origin of the radio jet in M87 at the location of the central black hole. Nature 477, 185-187 (2011).

25. Event Horizon Telescope Collaboration et al. First M87 event Horizon Telescope Results. I. The shadow of the supermassive black hole. Astrophys. J. Lett. 875, L1 (2019).

26. Walker, R. C., Hardee, P. E., Davies, F. B., Ly, C. \& Junor, W. The structure and dynamics of the subparsec jet in M87 based on 50 VLBA observations over 17 years at $43 \mathrm{GHz}$. Astrophys. J. 855, 128 (2018).

27. Kuo, C. Y. et al. Measuring mass accretion rate onto the supermassive black hole in M87 using Faraday rotation measure with the submillimeter array. Astrophys. J. Lett. 783, L33 (2014).

28. Abdo, A. A. et al. Fermi large area telescope view of the core of the radio galaxy Centaurus A. Astrophys. J. 719, 1433-1444 (2010).

29. Falcke, H. et al. The simultaneous spectrum of Sagittarius $A^{*}$ from 20 centimeters to 1 millimeter and the nature of the millimeter excess. Astrophys. J. 499, 731-734 (1998). 
30. Bower, G. C. et al. ALMA observations of the terahertz spectrum of Sagittarius A*. Astrophys. J. Lett. 881, L2 (2019).

31. Kim, J. et al. A VLBI receiving system for the south pole telescope. Proc. SPIE 10708, 107082S (2018)

32. Event Horizon Telescope Collaboration et al. First M87 Event Horizon Telescope results. II. Array and instrumentation. Astrophys. J. Lett. 875, L2 (2019).

33. Matthews, L. D. et al. The ALMA phasing system: a beamforming capability for ultra-high-resolution science at (sub)millimeter wavelengths. Publ. Astron. Soc. Pac. 130, 015002 (2018).

34. Whitney, A. R. et al. Demonstration of a 16 Gbps station broadband-RF VLBI system. Publ. Astron. Soc. Pac. 125, 196 (2013).

35. Deller, A. T., Tingay, S. J., Bailes, M. \& West, C. DiFX: a software correlator for very long baseline interferometry using multiprocessor computing environments. Publ. Astron. Soc. Pac. 119, 318-336 (2007).

36. Deller, A. T. et al. DiFX-2: a more flexible, efficient, robust, and powerful software correlator. Publ. Astron. Soc. Pac. 123, 275 (2011).

37. Martí-Vidal, I., Roy, A., Conway, J. \& Zensus, A. J. Calibration of mixed-polarization interferometric observations. Tools for the reduction of interferometric data from elements with linear and circular polarization receivers. Astron. Astrophys. 587, A143 (2016).

38. Goddi, C. et al. Calibration of ALMA as a phased array. ALMA observations during the 2017 VLBI campaign. Publ. Astron. Soc. Pac. 131, 075003 (2019)

39. Thompson, A. R., Moran, J. M. \& Swenson, G. W. J. Interferometry and Synthesis in Radio Astronomy 3rd edn (Springer, 2017).

40. Wootten, A. \& Thompson, A. R. The Atacama Large Millimeter/submillimeter Array. IEEE Proc. 97, 1463-1471 (2009).

41. Whitney, A. R. et al. Mark 4 VLBI correlator: architecture and algorithms. Radio Sci. 39, RS1007 (2004)

42. Schwab, F. R. \& Cotton, W. D. Global fringe search techniques for VLBI. Astron. J. 88, 688-694 (1983).

43. Alef, W. \& Porcas, R. W. VLBI fringe-fitting with antenna-based residuals. Astron. Astrophys. 168, 365-368 (1986).

44. Event Horizon Telescope Collaboration et al. First M87 Event Horizon Telescope results. III. Data processing and calibration. Astrophys. J. Lett. 875, L3 (2019).

45. Chael, A. A. et al. High-resolution linear polarimetric imaging for the Even Horizon Telescope. Astrophys. J. 829, 11 (2016).

46. Chael, A. A. et al. Interferometric imaging directly with closure phases and closure amplitudes. Astrophys. J. 857, 23 (2018).

47. Ojha, R. et al. TANAMI: tracking active galactic nuclei with austral milliarcsecond interferometry. I. First-epoch $8.4 \mathrm{GHz}$ images. Astron. Astrophys. 519, A45 (2010).

48. Kim, J. et al. The $1.4 \mathrm{~mm}$ core of Centaurus A: first VLBI results with the South Pole Telescope. Astrophys. J. 861, 129 (2018).

49. Event Horizon Telescope Collaboration et al. First M87 Event Horizon Telescope results. IV. Imaging the central supermassive black hole. Astrophys. J. Lett. 875, L4 (2019).

50. Akiyama, K. et al. Imaging the Schwarzschild-radius-scale structure of M87 with the Event Horizon Telescope using sparse modeling. Astrophys. J. 838, 1 (2017).

51. Akiyama, K. et al. Superresolution full-polarimetric imaging for radio interferometry with sparse modeling. Astron. J. 153, 159 (2017).

52. Shepherd, M. C., Pearson, T. J. \& Taylor, G. B. DIFMAP: an interactive program for synthesis imaging. Bull. Am. Astron. Soc. 26, 987-989 (1994).

53. Shepherd, M. C. Difmap: an interactive program for synthesis imaging. Astron. Soc. Pac. Conf. 125, 77 (1997).

54. Rau, U. \& Cornwell, T. J. A multi-scale multi-frequency deconvolution algorithm for synthesis imaging in radio interferometry. Astron. Astrophys. 532, A71 (2011)

55. Högbom, J. A. Aperture synthesis with a non-regular distribution of interferometer baselines. Astron. Astrophys. Suppl. Ser. 15, 417 (1974).

56. Clark, B. G. An efficient implementation of the algorithm 'CLEAN'. Astron. Astrophys. 89, 377 (1980)

57. Issaoun, S. et al. VLBI imaging of black holes via second moment regularization. Astron. Astrophys. 629, A32 (2019).

58. Blackburn, L. et al. Closure statistics in interferometric data. Astrophys. J. 894, 31 (2020).

59. Narayan, R. \& Nityananda, R. Maximum entropy image restoration in astronomy. Annu. Rev. Astron. Astrophys. 24, 127-170 (1986).

60. Roelofs, F. et al. SYMBA: an end-to-end VLBI synthetic data generation pipeline. Astron. Astrophys. 636, A5 (2020).

61. Pardo, J. R., Cernicharo, J. \& Serabyn, E. Atmospheric transmission at microwaves (ATM): an improved model for millimeter/submillimeter applications. IEEE Trans. Antennas Propag. 49, 1683-1694 (2001).

62. Blecher, T., Deane, R., Bernardi, G. \& Smirnov, O. MEQSILHOUETTE: a mm-VLBI observation and signal corruption simulator. Mon. Not. R. Astron. Soc. 464, 143-151 (2017)

63. Baczko, A.-K. et al. Asymmetric jet production in the active galactic nucleus of NGC 1052. Astron. Astrophys. 623, A27 (2019).
64. Nathanail, A. et al. Plasmoid formation in global GRMHD simulations and AGN flares. Mon. Not. R. Astron. Soc. 495, 1549-1565 (2020).

65. Pushkarev, A. B., Gabuzda, D. C., Vetukhnovskaya, Y. N. \& Yakimov, V. E. Spine-sheath polarization structures in four active galactic nuclei jets. Mon. Not. R. Astron. Soc. 356, 859-871 (2005).

66. Hovatta, T. et al. MOJAVE: monitoring of jets in active galactic nuclei with VLBA experiments. VIII. Faraday rotation in parsec-scale AGN jets. Astron. J. 144, 105 (2012)

67. Gabuzda, D. C., Nagle, M. \& Roche, N. The jets of AGN as giant coaxial cables. Astron. Astrophys. 612, A67 (2018)

68. Rybicki, G. B. \& Lightman, A. P. Radiative Processes in Astrophysics (Wiley, 1979).

69. Clausen-Brown, E., Lyutikov, M. \& Kharb, P. Signatures of large-scale magnetic fields in active galactic nuclei jets: transverse asymmetries. Mon. Not. R. Astron. Soc. 415, 2081-2092 (2011)

70. Boccardi, B. et al. The stratified two-sided jet of Cygnus A. Acceleration and collimation. Astron. Astrophys. 585, A33 (2016).

71. Boccardi, B., Krichbaum, T. P., Bach, U., Bremer, M. \& Zensus, J. A. First 3 $\mathrm{mm}$-VLBI imaging of the two-sided jet in Cygnus A. Zooming into the launching region. Astron. Astrophys. 588, L9 (2016).

72. Tadhunter, C. et al. Spectroscopy of the near-nuclear regions of Cygnus A: estimating the mass of the supermassive black hole. Mon. Not. R. Astron. Soc. 342, 861-875 (2003)

73. Pushkarev, A. B., Kovalev, Y. Y., Lister, M. L. \& Savolainen, T. MOJAVE-XIV. Shapes and opening angles of AGN jets. Mon. Not. R. Astron. Soc. 468 4992-5003 (2017).

74. Israel, F. P. Centaurus A-NGC 5128. Astron. Astrophys. Rev. 8, 237-278 (1998)

75. Hardcastle, M. J. et al. Radio and X-ray observations of the jet in Centaurus A. Astrophys. J. 593, 169-183 (2003).

76. Müller, C. et al. Dual-frequency VLBI study of Centaurus A on sub-parsec scales. The highest-resolution view of an extragalactic jet. Astron. Astrophys. 530, L11 (2011)

77. Silk, J. \& Rees, M. J. Quasars and galaxy formation. Astron. Astrophys. 331, L1-L4 (1998)

78. Magorrian, J. et al. The demography of massive dark objects in galaxy centers. Astron. J. 115, 2285-2305 (1998).

79. Pierre Auger Collaboration et al. An indication of anisotropy in arrival directions of ultra-high-energy cosmic rays through comparison to the flux pattern of extragalactic gamma-ray sources. Astrophys. J. Lett. 853, L29 (2018)

80. H.E.S.S. Collaboration et al. Resolving acceleration to very high energies along the jet of Centaurus A. Nature 582, 356-359 (2020).

81. Komissarov, S. S., Vlahakis, N., Königl, A. \& Barkov, M. V. Magnetic acceleration of ultrarelativistic jets in gamma-ray burst sources. Mon. Not. R. Astron. Soc. 394, 1182-1212 (2009).

82. Lyubarsky, Y. Asymptotic structure of poynting-dominated jets. Astrophys. J. 698, 1570-1589 (2009).

83. Falcke, H., Mannheim, K. \& Biermann, P. L. The Galactic Center radio jet. Astron. Astrophys. 278, L1-L4 (1993).

84. McKinney, J. C. General relativistic magnetohydrodynamic simulations of the jet formation and large-scale propagation from black hole accretion systems. Mon. Not. R. Astron. Soc. 368, 1561-1582 (2006).

85. Mościbrodzka, M., Falcke, H. \& Noble, S. Scale-invariant radio jets and varying black hole spin. Astron. Astrophys. 596, A13 (2016).

86. Davelaar, J. et al. Modeling non-thermal emission from the jet-launching region of M87 with adaptive mesh refinement. Astron. Astrophys. 632 A2 (2019).

87. Goddi, C. et al. Polarimetric properties of Event Horizon Telescope Targets from ALMA. Astrophys. J. Lett. 910, L14 (2021).

88. Rafferty, D. A., McNamara, B. R., Nulsen, P. E. J. \& Wise, M. W. The feedback-regulated growth of black holes and bulges through gas accretion and starbursts in cluster central dominant galaxies. Astrophys. J. 652, 216-231 (2006).

89. Sokolovsky, K. V., Kovalev, Y. Y., Pushkarev, A. B. \& Lobanov, A. P. A VLBA survey of the core shift effect in AGN jets. I. Evidence of dominating synchrotron opacity. Astron. Astrophys. 532, A38 (2011).

\section{Acknowledgements}

A.C. is an Einstein Fellow of the NASA Hubble Fellowship Program. J.P is an EACOA fellow. Z.Y. is a UKRI Stephen Hawking Fellow. We thank the following organizations and programmes: the Academy of Finland (projects 274477, 284495, 312496, 315721); the Agencia Nacional de Investigación y Desarrollo (ANID), Chile via NCN19_058 (TITANs) and Fondecyt 3190878; the Alexander von Humboldt Stiftung; an Alfred P. Sloan Research Fellowship; Allegro, the European ALMA Regional Centre node in the Netherlands, the NL astronomy research network NOVA and the astronomy institutes of the University of Amsterdam, Leiden University and Radboud University; the Black Hole Initiative at Harvard University, through a grant (60477) from the John Templeton Foundation; the China Scholarship Council; Consejo Nacional de Ciencia y Tecnología (CONACYT, Mexico, projects U0004-246083, U0004-259839, F0003-272050, M0037279006, F0003-281692, 104497, 275201, 263356, 57265507); the Delaney Family via 
the Delaney Family John A. Wheeler Chair at Perimeter Institute; Dirección General de Asuntos del Personal Académico-Universidad Nacional Autónoma de México (DGAPA-UNAM, projects IN112417 and IN112820); the EACOA Fellowship of the East Asia Core Observatories Association; the European Research Council Synergy Grant "BlackHoleCam: Imaging the Event Horizon of Black Holes" (grant 610058); the Generalitat Valenciana postdoctoral grant APOSTD/2018/177 and GenT Program (project CIDEGENT/2018/021); MICINN Research Project PID2019-108995GB-C22; the Gordon and Betty Moore Foundation (grants GBMF- 3561, GBMF-5278); the Istituto Nazionale di Fisica Nucleare (INFN) sezione di Napoli, iniziative specifiche TEONGRAV; the International Max Planck Research School for Astronomy and Astrophysics at the Universities of Bonn and Cologne; Joint Princeton/Flatiron and Joint Columbia/Flatiron Postdoctoral Fellowships, research at the Flatiron Institute is supported by the Simons Foundation; the Japanese Government (Monbukagakusho: MEXT) Scholarship; the Japan Society for the Promotion of Science (JSPS) Grant-in-Aid for JSPS Research Fellowship (JP17J08829); the Key Research Program of Frontier Sciences, Chinese Academy of Sciences (CAS, grants QYZDJ-SSW-SLH057, QYZDJSSWSYS008, ZDBS-LY-SLH011).

We further thank the Leverhulme Trust Early Career Research Fellowship; the Max-Planck-Gesellschaft (MPG); the Max Planck Partner Group of the MPG and the CAS; the MEXT/JSPS KAKENHI (grants 18KK0090, JP18K13594, JP18K03656, JP18H03721, 18K03709, 18H01245, JP19H01943, 25120007); the Malaysian Fundamental Research Grant Scheme (FRGS) FRGS/1/2019/STG02/UM/02/6; the MIT International Science and Technology Initiatives (MISTI) Funds; the Ministry of Science and Technology (MOST) of Taiwan (105- 2112-M-001-025-MY3, 1062112-M-001-011, 106-2119- M-001-027, 107-2119-M-001-017, 107-2119-M-001020, 107-2119-M-110-005, 108-2112-M-001-048, and 109-2124-M-001-005); the National Aeronautics and Space Administration (NASA, Fermi Guest Investigator grant 80NSSC20K1567, NASA Astrophysics Theory Program grant 80NSSC20K0527, NASA grant NNX17AL82G, and Hubble Fellowship grant HST-HF2-51431.001-A awarded by the Space Telescope Science Institute, which is operated by the Association of Universities for Research in Astronomy, Inc., for NASA, under contract NAS526555, and NASA NuSTAR award 80NSSC20K0645); the National Institute of Natural Sciences (NINS) of Japan; the National Key Research and Development Program of China (grant 2016YFA0400704, 2016YFA0400702); the National Science Foundation (NSF, grants AST-0096454, AST-0352953, AST-0521233, AST-0705062, AST-0905844, AST-0922984, AST-1126433, AST-1140030, DGE-1144085, AST-1207704, AST1207730, AST-1207752, MRI-1228509, OPP-1248097, AST-1310896, AST-1337663, AST-1440254, AST-1555365, AST-1615796, AST-1715061, AST-1716327, AST1716536, OISE-1743747, AST-1816420, AST-1903847, AST-1935980, AST-2034306); the Natural Science Foundation of China (grants 11573051, 11633006, 11650110427, 10625314, 11721303, 11725312, 11933007, 11991052, 11991053); a fellowship of China Postdoctoral Science Foundation (2020M671266); the Natural Sciences and Engineering Research Council of Canada (NSERC, including a Discovery Grant and the NSERC Alexander Graham Bell Canada Graduate Scholarships-Doctoral Program); the National Research Foundation of Korea (the Global PhD Fellowship Grant: grants 2014H1A2A1018695, NRF-2015H1A2A1033752, 2015- R1D1A1A01056807, the Korea Research Fellowship Program: NRF-2015H1D3A1066561, Basic Research Support Grant 2019R1F1A1059721); the Netherlands Organization for Scientific Research (NWO) VICI award (grant 639.043.513) and Spinoza Prize SPI 78-409; the New Scientific Frontiers with Precision Radio Interferometry Fellowship awarded by the South African Radio Astronomy Observatory (SARAO), which is a facility of the National Research Foundation (NRF), an agency of the Department of Science and Innovation (DSI) of South Africa; the South African Research Chairs Initiative of the Department of Science and Innovation and National Research Foundation; the Onsala Space Observatory (OSO) national infrastructure, for the provisioning of its facilities/observational support (OSO receives funding through the Swedish Research Council under grant 201700648) the Perimeter Institute for Theoretical Physics (research at Perimeter Institute is supported by the Government of Canada through the Department of Innovation, Science and Economic Development and by the Province of Ontario through the Ministry of Research, Innovation and Science); the Spanish Ministerio de Economía y Competitividad (grants PGC2018-098915-B-C21, AYA2016-80889-P, PID2019108995GB-C21); the State Agency for Research of the Spanish MCIU through the 'Center of Excellence Severo Ochoa' award for the Instituto de Astrofísica de Andalucía (SEV2017- 0709); the Toray Science Foundation; the Consejería de Economía, Conocimiento, Empresas y Universidad of the Junta de Andalucía (grant P18-FR-1769), the Consejo Superior de Investigaciones Cientificas (grant 2019AEP112); the US Department of Energy (US DOE) through the Los Alamos National Laboratory (operated by Triad National Security, LLC, for the National Nuclear Security Administration of the US DOE (Contract 89233218CNA000001); the European Union's Horizon 2020 research and innovation programme under grant agreement No 730562 RadioNet; ALMA North America Development Fund; the Academia Sinica; Chandra TM6- 17006X and DD718089X; the GenT Program (Generalitat Valenciana) Project CIDEGENT/2018/021.

This work used the Extreme Science and Engineering Discovery Environment (XSEDE), supported by NSF grant ACI-1548562, and CyVerse, supported by NSF grants DBI-0735191, DBI-1265383, and DBI-1743442. XSEDE Stampede2 resource at TACC was allocated through TG-AST170024 and TG-AST080026N. XSEDE JetStream resource at PTI and TACC was allocated through AST170028. The simulations were performed in part on the SuperMUC cluster at the LRZ in Garching, on the LOEWE cluster in CSC in Frankfurt, and on the HazelHen cluster at the HLRS in Stuttgart. This research was enabled in part by support provided by Compute Ontario (http://computeontario. ca), Calcul Quebec (http://www.calculquebec.ca) and Compute Canada (http://www. computecanada.ca). We thank the staff at the participating observatories, correlation centres, and institutions for their enthusiastic support.

This paper makes use of the following ALMA data: ADS/JAO.ALMA\#2016.1.01198.V. ALMA is a partnership of the European Southern Observatory (ESO; Europe, representing its member states), NSF, and National Institutes of Natural Sciences of Japan, together with National Research Council (Canada), Ministry of Science and Technology (MOST; Taiwan), Academia Sinica Institute of Astronomy and Astrophysics (ASIAA; Taiwan), and Korea Astronomy and Space Science Institute (KASI; Republic of Korea), in cooperation with the Republic of Chile. The Joint ALMA Observatory is operated by ESO, Associated Universities, Inc. (AUI)/NRAO, and the National Astronomical Observatory of Japan (NAOJ). The NRAO is a facility of the NSF operated under cooperative agreement by AUI. APEX is a collaboration between the Max-Planck-Institut für Radioastronomie (Germany), ESO, and the Onsala Space Observatory (Sweden). The SMA is a joint project between the SAO and ASIAA and is funded by the Smithsonian Institution and the Academia Sinica. The JCMT is operated by the East Asian Observatory on behalf of the NAOJ, ASIAA, and KASI, as well as the Ministry of Finance of China, Chinese Academy of Sciences, and the National Key R\&D Program (No. 2017YFA0402700) of China. Additional funding support for the JCMT is provided by the Science and Technologies Facility Council (UK) and participating universities in the UK and Canada. The LMT is a project operated by the Instituto Nacional de Astrófisica, Óptica, y Electrónica (Mexico) and the University of Massachusetts at Amherst (USA), with financial support from the Consejo Nacional de Ciencia y Tecnología and the National Science Foundation. The IRAM 30-m telescope on Pico Veleta, Spain is operated by IRAM and supported by CNRS (Centre National de la Recherche Scientifique, France), MPG (Max-PlanckGesellschaft, Germany) and IGN (Instituto Geográfico Nacional, Spain). The SMT is operated by the Arizona Radio Observatory, a part of the Steward Observatory of the University of Arizona, with financial support of operations from the State of Arizona and financial support for instrumentation development from the NSF. The SPT is supported by the National Science Foundation through grant PLR- 1248097. Partial support is also provided by the NSF Physics Frontier Center grant PHY-1125897 to the Kavli Institute of Cosmological Physics at the University of Chicago, the Kavli Foundation and the Gordon and Betty Moore Foundation grant GBMF 947. The SPT hydrogen maser was provided on loan from the GLT, courtesy of ASIAA. The EHTC has received generous donations of FPGA chips from Xilinx Inc., under the Xilinx University Program. The EHTC has benefited from technology shared under open-source license by the Collaboration for Astronomy Signal Processing and Electronics Research (CASPER). The EHT project is grateful to T4Science and Microsemi for their assistance with Hydrogen Masers. This research has made use of NASA's Astrophysics Data System. We gratefully acknowledge the support provided by the extended staff of the ALMA, both from the inception of the ALMA Phasing Project through the observational campaigns of 2017 and 2018. We would like to thank A. Deller and W. Brisken for EHT-specific support with the use of DiFX. We acknowledge the significance that Maunakea, where the SMA and JCMT EHT stations are located, has for the indigenous Hawaiian people.

The grants listed above collectively fund the Event Horizon Telescope project.

\section{Author contributions}

K.A., L.B., C.-k.C., S.I., M.J., J.K., J.Y.K., T.P.K., J.L., E.L., D.P.M., V.R., K.L.J.R., I.V.B. and M.W. have worked on the calibration of the EHT data. K.A., K.L.B., A.C., J.L.G., S.I., M.J., M.D.J., C.N., D.W.P., F.R. and M.W. have worked on the image reconstruction. M.B., K.C., J.D., P.G.E., H.F., C.M.F., C.G., M.J., M.K., Y.M., A.M., S.M., E.R. and M.W. have worked on the interpretation of the results. M.J., M.K., C.M. and E.R. have coordinated the research. The Event Horizon Telescope collaboration as a whole has enabled this research by building the EHT instrument and producing the tools and knowledge for the reduction, analysis and interpretation of the data.

\section{Funding}

Open access funding provided by Max Planck Institute for Radio Astronomy (2)

\section{Competing interests}

The authors declare no competing interests.

\section{Additional information}

Supplementary information The online version contains supplementary material available at https://doi.org/10.1038/s41550-021-01417-w.

\section{Correspondence and requests for materials should be addressed to M.J.}

Peer review information Nature Astronomy thanks Denise Gabuzda, Talvikki Hovatta and the other, anonymous, reviewer(s) for their contribution to the peer review of this work.

Reprints and permissions information is available at www.nature.com/reprints.

Publisher's note Springer Nature remains neutral with regard to jurisdictional claims in

published maps and institutional affiliations.

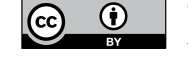

Open Access This article is licensed under a Creative Commons Attribution 4.0 International License, which permits use, sharing, adaptation, distribution and reproduction in any medium or format, as long as you give appropriate credit to the original author(s) and the source, provide a link to 
the Creative Commons license, and indicate if changes were made. The images or other third party material in this article are included in the article's Creative Commons license, unless indicated otherwise in a credit line to the material. If material is not included in the article's Creative Commons license and your intended use is not permitted by statu- tory regulation or exceeds the permitted use, you will need to obtain permission directly from the copyright holder. To view a copy of this license, visit http://creativecommons. org $/$ licenses/by/4.0/.

(c) The Author(s) 2021

\section{The Event Horizon Telescope Collaboration}

Kazunori Akiyama 4,6,7, Antxon Alberdi ${ }^{19}$, Walter Alef', Juan Carlos Algaba ${ }^{30}$, Richard Anantua ${ }^{4,5,16}$, Keiichi Asada ${ }^{21}$, Rebecca Azulay 1,31,32, Anne-Kathrin Baczko', David Ball'2, Mislav Baloković8,9, John Barrett ${ }^{6}$, Bradford A. Benson ${ }^{33,34}$, Dan Bintley ${ }^{35}$, Lindy Blackburn ${ }^{4,5}$, Raymond Blundell ${ }^{5}$, Wilfred Boland ${ }^{36}$, Katherine L. Bouman ${ }^{4,5,10}$, Geoffrey C. Bower ${ }^{37}$, Hope Boyce ${ }^{38,39}$, Michael Bremer ${ }^{40}$, Christiaan D. Brinkerink2 ${ }^{2}$, Roger Brissenden ${ }^{4,5}$, Silke Britzen', Avery E. Broderick ${ }^{26,27,41}$, Dominique Broguiere ${ }^{40}$, Thomas Bronzwaer2 ${ }^{2}$ Do-Young Byun ${ }^{42,43}$, John E. Carlstrom $34,44,45,46$, Andrew Chael ${ }^{11}$, Chi-kwan Chan ${ }^{12,13}$, Koushik Chatterjee ${ }^{14}$, Shami Chatterjee ${ }^{47}$, Ming-Tang Chen ${ }^{37}$, Yongjun Chen ${ }^{48,49}$, Paul M. Chesler ${ }^{4}$, Ilje Cho ${ }^{42,43}$, Pierre Christian ${ }^{50}$, John E. Conway ${ }^{51}$, James M. Cordes ${ }^{47}$, Thomas M. Crawford ${ }^{34,44}$, Geoffrey B. Crew ${ }^{6}$, Alejandro Cruz-Osorio ${ }^{18}$, Yuzhu Cui ${ }^{52,53}$, Jordy Davelaar ${ }^{2,15,16}$, Mariafelicia De Laurentis ${ }^{18,54,55}$, Roger Deane ${ }^{56,57,58}$, Jessica Dempsey ${ }^{35}$, Gregory Desvignes ${ }^{59}$, Jason Dexter ${ }^{60}$, Sheperd S. Doeleman ${ }^{4,5}$, Ralph P. Eatough ${ }^{1,61}$, Joseph Farah ${ }^{4,5,62}$, Heino Falcke², Vincent L. Fish ${ }^{6}$, Ed Fomalont ${ }^{63}$, H. Alyson Ford ${ }^{12}$, Raquel Fraga-Encinas ${ }^{2}$, Per Friberg ${ }^{35}$, Christian M. Fromm ${ }^{4,5,18}$, Antonio Fuentes ${ }^{19}$, Peter Galison ${ }^{4,64,65}$, Charles F. Gammie ${ }^{66,67}$, Roberto García ${ }^{40}$, Zachary Gelles ${ }^{4,5}$, Olivier Gentaz ${ }^{40}$, Boris Georgiev ${ }^{26,27}$, Ciriaco Goddi ${ }^{2,20}$, Roman Gold41,68, José L. Gómez ${ }^{19}$, Arturo I. Gómez-Ruiz ${ }^{69,70}$, Minfeng Gu4,71, Mark Gurwell ${ }^{5}$, Kazuhiro Hada ${ }^{52,53}$, Daryl Haggard ${ }^{38,39}$, Michael H. Hecht ${ }^{6}$, Ronald Hesper ${ }^{72}$, Elizabeth Himwich ${ }^{4,73}$, Luis C. Ho ${ }^{74,75}$, Paul Ho ${ }^{21}$, Mareki Honma ${ }^{52,53,76}$, Chih-Wei L. Huang ${ }^{21}$, Lei Huang ${ }^{48,71}$, David H. Hughes ${ }^{69}$, Shiro Ikeda7,77,78,79, Makoto Inoue ${ }^{21}$, Sara Issaoun ${ }^{2}$, David J. James ${ }^{4,5}$, Buell T. Jannuzi ${ }^{12}$, Michael Janssen ${ }^{1,2}$, Britton Jeter ${ }^{26,27}$, Wu Jiang ${ }^{48}$, Alejandra Jimenez-Rosales ${ }^{2}$, Michael D. Johnson ${ }^{4,5}$, Svetlana Jorstad ${ }^{80,81}$, Taehyun Jung ${ }^{42,43}$, Mansour Karami26,41, Ramesh Karuppusamy', Tomohisa Kawashima ${ }^{82}$, Garrett K. Keating ${ }^{5}$, Mark Kettenis ${ }^{29}$, Dong-Jin Kim', Jae-Young Kim ${ }^{1,42}$, Junhan Kim ${ }^{10,12}$, Jongsoo Kim ${ }^{42}$, Motoki Kino ${ }^{7,83}$, Jun Yi Koay21, Yutaro Kofuji52,76, Shoko Koyama ${ }^{21}$, Michael Kramer', Carsten Kramer ${ }^{40}$, Thomas P. Krichbaum ${ }^{1}$, Cheng-Yu Kuo ${ }^{21,84}$, Tod R. Lauer ${ }^{85}$, Sang-Sung Lee ${ }^{42}$, Aviad Levis ${ }^{10}$, Yan-Rong Li ${ }^{86}$, Zhiyuan Li ${ }^{87,88}$, Michael Lindqvist ${ }^{51}$, Rocco Lico,19, Greg Lindahl5, Jun Liu', Kuo Liu', Elisabetta Liuzzo ${ }^{22}$, Wen-Ping Lo ${ }^{21,89}$, Andrei P. Lobanov1, Laurent Loinard ${ }^{90,91}$, Colin Lonsdale ${ }^{6}$, Ru-Sen Lu' ${ }^{1,48,49}$, Nicholas R. MacDonald', Jirong Mao ${ }^{92,93,94}$, Nicola Marchili1,22, Sera Markoff ${ }^{14,23}$, Daniel P. Marrone ${ }^{12}$, Alan P. Marscher ${ }^{80}$, Iván Martí-Vidal' ${ }^{31,32}$, Satoki Matsushita ${ }^{21}$, Lynn D. Matthews ${ }^{6}$, Lia Medeiros ${ }^{12,95}$, Karl M. Menten', Izumi Mizuno ${ }^{35}$, Yosuke Mizuno ${ }^{18,25}$, James M. Moran ${ }^{4,5}$, Kotaro Moriyama,52, Monika Moscibrodzka², Cornelia Müller,2, Gibwa Musoke 2,14, Alejandro Mus Mejías ${ }^{31,32}$, Hiroshi Nagai, ${ }^{73}$, Neil M. Nagar ${ }^{28}$, Masanori Nakamura ${ }^{21,96}$, Ramesh Narayan 4,5, Gopal Narayanan ${ }^{97}$, Iniyan Natarajan ${ }^{56,58,98,}$ Antonios Nathanail ${ }^{18,99}$, Joey Neilsen ${ }^{100}$, Roberto Neri ${ }^{40}$, Chunchong Ni26,27, Aristeidis Noutsos ${ }^{1}$, Michael A. Nowak ${ }^{101}$, Hiroki Okino52,76, Héctor Olivares², Gisela N. Ortiz-León', Tomoaki Oyama52, Feryal Özel ${ }^{12}$, Daniel C. M. Palumbo ${ }^{4,5}$, Jongho Park ${ }^{21}$, Nimesh Patel ${ }^{5}$, Ue-Li Pen ${ }^{41,102,103,104}$, Dominic W. Pesce ${ }^{4,5}$, Vincent Piétu ${ }^{40}$, Richard Plambeck ${ }^{105}$, Aleksandar PopStefanija97, Oliver Porth ${ }^{14,18}$, Felix M. Pötzl', Ben Prather ${ }^{66}$, Jorge A. Preciado-López ${ }^{41}$, Dimitrios Psaltis ${ }^{12}$, Hung-Yi Pu ${ }^{21,41,106}$, Venkatessh Ramakrishnan ${ }^{28}$, Ramprasad Rao ${ }^{37}$, Mark G. Rawlings ${ }^{35}$, 
Alexander W. Raymond 4,5, Luciano Rezzolla ${ }^{18,107,108}$, Angelo Ricarte ${ }^{4,5}$, Bart Ripperda ${ }^{16,109}$, Freek Roelofs ${ }^{2,5}$, Alan Rogers ${ }^{6}$, Eduardo Ros ${ }^{1}$, Mel Rose $^{12}$, Arash Roshanineshat ${ }^{12}$, Helge Rottmann', Alan L. Roy ${ }^{1}$, Chet Ruszczyk ${ }^{6}$, Kazi L. J. Rygl ${ }^{22}$, Salvador Sánchez ${ }^{110}$, David Sánchez-Arguelles ${ }^{69,70}$, Mahito Sasada ${ }^{52,111}$, Tuomas Savolainen ${ }^{1,112,113}$, F. Peter Schloerb ${ }^{97}$, Karl-Friedrich Schuster ${ }^{40}$, Lijing Shao ${ }^{1,75}$, Zhiqiang Shen ${ }^{48,49}$, Des Small29, Bong Won Sohn ${ }^{42,43,114}$, Jason SooHoo6, He Sun ${ }^{10}$, Fumie Tazaki ${ }^{52}$, Alexandra J. Tetarenko ${ }^{35}$, Paul Tiede ${ }^{26,27}$, Remo P. J. Tilanus ${ }^{2,12,20,115}$, Michael Titus ${ }^{6}$, Pablo Torne ${ }^{1,110}$, Tyler Trent ${ }^{12}$, Efthalia Traianou', Sascha Trippe ${ }^{116}$, Ilse van Bemmel ${ }^{29}$, Huib Jan van Langevelde ${ }^{29,117}$, Daniel R. van Rossum², Jan Wagner ${ }^{1}$, Derek Ward-Thompson ${ }^{118}$, John Wardle ${ }^{119}$, Jonathan Weintroub ${ }^{4,5}$, Norbert Wex', Robert Wharton', Maciek Wielgus ${ }^{4,5}$, George N. Wong ${ }^{66}$, Qingwen Wu' ${ }^{120}$, Doosoo Yoon ${ }^{14}$, André Young ${ }^{2}$, Ken Young ${ }^{5}$, Ziri Younsi ${ }^{18,121}$, Feng Yuan ${ }^{48,71,122}$, Ye-Fei Yuan ${ }^{123}$, J. Anton Zensus ${ }^{1}$, Guang-Yao Zhao ${ }^{19}$ and Shan-Shan Zhao ${ }^{48}$

${ }^{30}$ Department of Physics, Faculty of Science, University of Malaya, Kuala Lumpur, Malaysia. ${ }^{31}$ Departament d'Astronomia i Astrofísica, Universitat de València, Valencia, Spain. ${ }^{32}$ Observatori Astronòmic, Universitat de València, Valencia, Spain. ${ }^{33}$ Fermi National Accelerator Laboratory, Batavia, IL, USA. ${ }^{34}$ Department of Astronomy and Astrophysics, University of Chicago, Chicago, IL, USA. ${ }^{35}$ East Asian Observatory, Hilo, HI, USA. ${ }^{36}$ Nederlandse Onderzoekschool voor Astronomie (NOVA), Leiden, The Netherlands. ${ }^{37}$ Institute of Astronomy and Astrophysics, Academia Sinica, Hilo, HI, USA. ${ }^{38}$ Department of Physics, McGill University, Montreal, Quebec, Canada. ${ }^{39}$ McGill Space Institute, McGill University, Montreal, Quebec, Canada. ${ }^{40}$ Institut de Radioastronomie Millimétrique, Saint Martin d'Hères, France. ${ }^{41}$ Perimeter Institute for Theoretical Physics, Waterloo, Ontario, Canada. ${ }^{42}$ Korea Astronomy and Space Science Institute, Daejeon, Republic of Korea. ${ }^{43}$ University of Science and Technology, Daejeon, Republic of Korea. ${ }^{44 K a v l i ~ I n s t i t u t e ~}$ for Cosmological Physics, University of Chicago, Chicago, IL, USA. ${ }^{45}$ Department of Physics, University of Chicago, Chicago, IL, USA. ${ }^{46}$ Enrico Fermi Institute, University of Chicago, Chicago, IL, USA. ${ }^{47}$ Cornell Center for Astrophysics and Planetary Science, Cornell University, Ithaca, NY, USA. ${ }^{48}$ Shanghai Astronomical Observatory, Chinese Academy of Sciences, Shanghai, People's Republic of China. ${ }^{49}$ Key Laboratory of Radio Astronomy, Chinese Academy of Sciences, Nanjing, People's Republic of China. ${ }^{50}$ Physics Department, Fairfield University, Fairfield, CT, USA. ${ }^{51}$ Department of Space, Earth and Environment, Chalmers University of Technology, Onsala Space Observatory, Onsala, Sweden. ${ }^{52}$ Mizusawa VLBI Observatory, National Astronomical Observatory of Japan, Oshu, Japan. ${ }^{53}$ Department of Astronomical Science, The Graduate University for Advanced Studies (SOKENDAI), Mitaka, Japan. ${ }^{54}$ Dipartimento di Fisica "E. Pancini", Universitá di Napoli "Federico II", Complesso Universitario di Monte Sant'Angelo, Naples, Italy. ${ }^{55}$ INFN Sezione di Napoli, Complesso Universitario di Monte Sant'Angelo, Naples, Italy. ${ }^{56}$ Wits Centre for Astrophysics, University of the Witwatersrand, Johannesburg, South Africa. ${ }^{57}$ Department of Physics, University of Pretoria, Pretoria, South Africa. ${ }^{58} \mathrm{Centre}$ for Radio Astronomy Techniques and Technologies, Department of Physics and Electronics, Rhodes University, Makhanda, South Africa. ${ }^{59}$ LESIA, Observatoire de Paris, Université PSL, CNRS, Sorbonne Université, Université de Paris, Meudon, France. ${ }^{60} \mathrm{JILA}$ and Department of Astrophysical and Planetary Sciences, University of Colorado, Boulder, CO, USA ${ }^{61}$ National Astronomical Observatories, Chinese Academy of Sciences, Beijing, People's Republic of China. ${ }^{62}$ University of Massachusetts Boston, Boston, MA, USA. ${ }^{63}$ National Radio Astronomy Observatory, Charlottesville, VA, USA. ${ }^{64}$ Department of History of Science, Harvard University, Cambridge, MA, USA. ${ }^{65}$ Department of Physics, Harvard University, Cambridge, MA, USA. ${ }^{66}$ Department of Physics, University of IIlinois, Urbana, IL, USA. ${ }^{67}$ Department of Astronomy, University of Illinois at Urbana-Champaign, Urbana, IL, USA. ${ }^{68} \mathrm{CP} 3-$ Origins, University of Southern Denmark, Odense, Denmark. ${ }^{69}$ Instituto Nacional de Astrofísica, Puebla, Mexico. ${ }^{70}$ Consejo Nacional de Ciencia y Tecnología, Mexico City, Mexico. ${ }^{71}$ Key Laboratory for Research in Galaxies and Cosmology, Chinese Academy of Sciences, Shanghai, People's Republic of China. ${ }^{72}$ NOVA Sub-mm Instrumentation Group, Kapteyn Astronomical Institute, University of Groningen, Groningen, The Netherlands. ${ }^{73}$ Center for the Fundamental Laws of Nature, Harvard University, Cambridge, MA, USA. ${ }^{74}$ Department of Astronomy, School of Physics, Peking University, Beijing, People's Republic of China. ${ }^{75}$ Kavli Institute for Astronomy and Astrophysics, Peking University, Beijing, People's Republic of China. ${ }^{76}$ Department of Astronomy, Graduate School of Science, The University of Tokyo, Bunkyo-ku, Japan. ${ }^{77} T$ The Institute of Statistical Mathematics, Tachikawa, Japan. ${ }^{78}$ Department of Statistical Science, The Graduate University for Advanced Studies (SOKENDAI), Tachikawa, Japan. ${ }^{79} \mathrm{Kavli}$ Institute for the Physics and Mathematics of the Universe, The University of Tokyo, Kashiwa, Japan. ${ }^{80}$ Institute for Astrophysical Research, Boston University, Boston, MA, USA. ${ }^{81}$ Astronomical Institute, St. Petersburg University, St. Petersburg, Russia. ${ }^{82}$ Institute for Cosmic Ray Research, The University of Tokyo, Kashiwa, Japan. ${ }^{83}$ Kogakuin University of Technology and Engineering, Academic Support Center, Hachioji, Japan. ${ }^{84}$ Physics Department, National Sun Yat-Sen University, Kaosiung City, Taiwan, ROC. ${ }^{85}$ National Optical Astronomy Observatory, Tucson, AZ, USA. ${ }^{86}$ Key Laboratory for Particle Astrophysics, Institute of High Energy Physics, Chinese Academy of Sciences, Beijing, People's Republic of China. ${ }^{87}$ School of Astronomy and Space Science, Nanjing University, Nanjing, People's Republic of China. ${ }^{88}$ Key Laboratory of Modern Astronomy and Astrophysics, Nanjing University, Nanjing, People's Republic of China. ${ }^{89}$ Department of Physics, National Taiwan University, Taipei, Taiwan, ROC. ${ }^{90}$ Instituto de Radioastronomía y Astrofísica, Universidad Nacional Autónoma de México, Morelia, Mexico. ${ }^{91}$ Instituto de Astronomía, Universidad Nacional Autónoma de México, Mexico City, Mexico. ${ }^{92}$ Yunnan Observatories, Chinese Academy of Sciences, Kunming, People's Republic of China. ${ }^{93}$ Center for Astronomical Mega-Science, Chinese Academy of Sciences, Beijing, People's Republic of China. ${ }^{94} \mathrm{Key}$ Laboratory for the Structure and Evolution of Celestial Objects, Chinese Academy of Sciences, Kunming, People's Republic of China. ${ }^{95}$ School of Natural Sciences, Institute for Advanced Study, Princeton, NJ, USA. ${ }^{96} \mathrm{National}$ Institute of Technology, Hachinohe College, Hachinohe City, Japan. ${ }^{97}$ Department of Astronomy, University of Massachusetts, Amherst, MA, USA. ${ }^{98}$ South African Radio Astronomy Observatory, Cape Town, South Africa. ${ }^{99}$ Department of Physics, National and Kapodistrian University of Athens, Zografos, Greece. ${ }^{100}$ Villanova University, Villanova, PA, USA. ${ }^{101}$ Physics Department, Washington University, St Louis, MO, USA. ${ }^{102}$ Canadian Institute for Theoretical Astrophysics, University of Toronto, Toronto, Ontario, Canada. ${ }^{103}$ Dunlap Institute for Astronomy and Astrophysics, University of Toronto, Toronto, Ontario, Canada. ${ }^{104}$ Canadian Institute for Advanced Research, Toronto, Ontario, Canada. ${ }^{105}$ Radio Astronomy Laboratory, University of California, Berkeley, CA, USA. ${ }^{106}$ Department of Physics, National Taiwan Normal University, Taipei, Taiwan, ROC. ${ }^{107}$ Frankfurt Institute for Advanced Studies, Frankfurt, Germany. ${ }^{108}$ School of Mathematics, Trinity College, Dublin, Ireland. ${ }^{109}$ Department of Astrophysical Sciences, Peyton Hall, Princeton University, Princeton, NJ, USA. ${ }^{110}$ Instituto de Radioastronomía Milimétrica, IRAM, Granada, Spain. ${ }^{11}$ Hiroshima Astrophysical Science Center, Hiroshima University, Higashi-Hiroshima, Japan. ${ }^{12}$ Department of Electronics and Nanoengineering, Aalto University, Aalto, Finland. ${ }^{113}$ Metsähovi Radio Observatory, Aalto University, Kylmälä, Finland. ${ }^{114}$ Department of Astronomy, Yonsei University, Seoul, Republic of Korea. ${ }^{115}$ Netherlands Organisation for Scientific Research (NWO), Den Haag, The Netherlands. ${ }^{116}$ Department of Physics and Astronomy, Seoul National University, Seoul, Republic of Korea. ${ }^{177}$ Leiden Observatory, Leiden University, 
Leiden, The Netherlands. ${ }^{118}$ Jeremiah Horrocks Institute, University of Central Lancashire, Preston, UK. ${ }^{119}$ Physics Department, Brandeis University, Waltham, MA, USA. ${ }^{120}$ School of Physics, Huazhong University of Science and Technology, Wuhan, Hubei, People's Republic of China. ${ }^{121}$ Mullard Space Science Laboratory, University College London, Surrey, UK. ${ }^{122}$ School of Astronomy and Space Sciences, University of Chinese Academy of Sciences, Beijing, People's Republic of China. ${ }^{123}$ Astronomy Department, University of Science and Technology of China, Hefei, People's Republic of China. 